\title{
The variational formulas of the volume function in the equiaffine geometry
}

\author{
Naoyuki KoIKe \\ (Received January 11, 2005; Revised September 16, 2005)
}

\begin{abstract}
We obtain the first and second variational formulas for the volume function over the space of all equiaffine immersions. By using the first variational formula, we define some kinds of volume functions over the space of all non-degenerate immersions and obtain the Euler-Lagrange equation for those volume functions. Also, in the case where the ambient space is flat, we obtain the second variational formulas for special ones of those volume functions.

Key words: equiaffine immersion, non-degenerate immersion, Tchebychev tensor field, volume ratio function.
\end{abstract}

\section{Introduction}

Let $D$ be a relatively compact domain with smooth boundary of an $n$ dimensional manifold $M$. Let $f$ be a non-degenerate immersion of $M$ into the $(n+1)$-dimensional equiaffine space $\left(\mathbf{R}^{n+1}, \widetilde{\nabla}, \widetilde{\omega}\right)$, where $\widetilde{\nabla}$ is the affine connection defined by $\widetilde{\nabla}_{\partial / \partial x^{i}} \partial / \partial x^{j}=0(1 \leq i, j \leq n+1)$ and $\widetilde{\omega}$ is the volume element defined by $\widetilde{\omega}\left(\partial / \partial x^{1}, \ldots, \partial / \partial x^{n+1}\right)=1$ in terms of the natural coordinate $\left(x^{1}, \ldots, x^{n+1}\right)$ of $\mathbf{R}^{n+1}$. The immersion $f$ admits uniquely a pair $\left(N, \theta^{\perp}\right)$ of its transversal bundle $N$ and its transversal volume element $\theta^{\perp}$ such that $\left(f, N, \theta^{\perp}\right)$ is an equiaffine immersion with $\theta=\omega_{h}$ (i.e., a Blaschke immersion), where $\theta$ is the induced volume element and $\omega_{h}$ is the volume element determined by the affine fundamental form $h$. The volume element $\theta$ is called the affine volume element. In 1989, L. Verstraelen and L. Vrancken $[\mathrm{VeVr}]$ obtained the first and second variational formulas for the volume function $\mathrm{Vol}$ (for the affine volume element) over the whole of the restriction $\left.f\right|_{D}$ to $D$ of a non-degenerate immersion $f$ of $M$ into the equiaffine space $\left(\mathbf{R}^{n+1}, \widetilde{\nabla}, \widetilde{\omega}\right)$ satisfying $\left.f\right|_{M \backslash D}=\phi$, where $\phi$ is a fixed nondegenerate immersion of $M \backslash D$ into $\left(\mathbf{R}^{n+1}, \widetilde{\nabla}, \widetilde{\omega}\right)$. Note that they treat only transversal variations. They showed that the affine mean curvature of $f$ vanishes on $D$ if and only if $\left.\frac{d}{d t}\right|_{t=0} \operatorname{Vol}\left(\left.f_{t}\right|_{D}\right)=0$ for any transversal

2000 Mathematics Subject Classification : 53A15. 
variation $f_{t}$ of $f\left(f_{0}=f\right)$ consisting of non-degenerate immersions with $\left.f_{t}\right|_{M \backslash D}=\phi$.

Remark 1.1 In 1982, E. Calabi [C] has already obtained the first and second variational formulas for the same volume function over the whole of locally strongly convex immersion into $\left(\mathbf{R}^{n+1}, \widetilde{\nabla}, \widetilde{\omega}\right)$.

In 2002, M. Wiehe [Wi] treated regular (non-degenerate in the sense of this paper) immersions of general codimension into the equiaffine space $\left(\mathbf{R}^{n+r}, \widetilde{\nabla}, \widetilde{\omega}\right)$. Such an immersion $f$ admits uniquely a pair $\left(N, \theta^{\perp}\right)$ of its transversal bundle $N$ and its transversal volume element $\theta^{\perp}$ such that $\left(f, N, \theta^{\perp}\right)$ is an equiaffine immersion with $\theta=\omega_{h}$ and $T=0$, where $\theta$ is the induced volume element, $\omega_{h}$ is the volume element defined from the affine fundamental form $h$ and $T$ is the Tchebychev tensor field. We call the volume element $\theta$ the affine volume element. Such an equiaffine immersion is interpreted as the higher codimension version of a Blaschke immersion. Hence we call such an immersion a Blaschke immersion of general codimension. He obtained the first variational formula for the volume function Vol (for the affine volume element) over the whole of the restriction $\left.f\right|_{D}$ to $D$ of such an immersion $f$ of $M$ into $\left(\mathbf{R}^{n+r}, \widetilde{\nabla}, \widetilde{\omega}\right)$ satisfying $\left.f\right|_{M \backslash D}=\phi$, where $D$ and $\phi$ are as above. He showed that the affine mean curvature function of such an immersion $f$ vanishes on $D$ if and only if $\left.\frac{d}{d t}\right|_{t=0} \operatorname{Vol}\left(\left.f_{t}\right|_{D}\right)=0$ holds for any variation $f_{t}$ of $f\left(f_{0}=f\right)$ consisting of such immersions.

Remark 1.2 In 1994, F. Dillen, G. Mys, L. Verstraelen and L. Vrancken [DMVV] treated certain kind of equiaffine immersions of codimension two into $\left(\mathbf{R}^{4}, \widetilde{\nabla}, \widetilde{\omega}\right)$. It is shown that the equiaffine immersions are Blaschke immersion of codimension two. They obtained the first variational formula for a certain kind of volume function over the whole of such immersions. It is shown that the volume function coincides with one for the affine volume element. Thus the result of [Wi] includes that of [DMVV].

In this paper, we treat general equiaffine immersions into a general equiaffine manifold. For each immersion $f$ of a manifold $M$ into an equiaffine manifold $(\widetilde{M}, \widetilde{\nabla}, \widetilde{\theta})$, there exist infinitely many pairs $\left(N, \theta^{\perp}\right)$ of its transversal bundle $N$ and its transversal volume element $\theta^{\perp}$ such that $\left(f, N, \theta^{\perp}\right)$ is an equiaffine immersion. To each equiaffine immersion $\left(f, N, \theta^{\perp}\right)$, an immersion of $M$ into the exterior product bundle $\bigwedge^{r}(T \widetilde{M})(r:=\operatorname{dim} \widetilde{M}-$ $\operatorname{dim} M)$ of degree $r$ of the tangent bundle $T \widetilde{M}$ satisfying some conditions 
corresponds bijectively (see Section 2). Hence we can define an equiaffine immersion into $(\widetilde{M}, \widetilde{\nabla}, \widetilde{\theta})$ as such an immersion into $\bigwedge^{r}(T \widetilde{M})$. When we consider the variational problem of equiaffine immersions, we had better define an equiaffine immersion as such an immersion into $\bigwedge^{r}(T \widetilde{M})$ rather than the triple $\left(f, N, \theta^{\perp}\right)$ because the variation vector field is then catched as a vector field along the immersion. Hence we shall define an equiaffine immersion as such an immersion. We first obtain the first and second variational formulas for the volume function over the space of all equiaffine immersions (see Theorem 3.1). Next we investigate some classes consisting of non-degenerate equiaffine immersions (see Theorem 4.1) and obtain the first variational formulas for the volume functions over the classes (see Theorem 5.1). According to the first variational formula, some volume functions are well-defined over the space of all non-degenerate immersions. We obtain the Euler-Lagrange equations for those volume functions (see Theorem 5.4). In particular, we can obtain the Euler-Lagrange equation for the volume function over the space of all $c$-Blaschke immersions. See Section 4 about the notion of a $c$-Blaschke immersion. In the case where the ambient space is flat, the Euler-Lagrange equation is reduced to $H=0$ ( $H$ : the affine mean curvature). Furthermore, we obtain the second variational formula for the volume function over the space of all $c$-Blaschke immersions into a flat equiaffine space (see Theorem 5.7).

\section{The definition of an equiaffine immersion in a new style}

In $[\mathrm{K}]$, we defined the notion of an equiaffine immersion of general codimension. We consider a variation of the immersions. For its purpose, we had better define the notion in a new style as follows. Let $(\widetilde{M}, \widetilde{\nabla}, \widetilde{\theta})$ be an $(n+r)$-dimensional equiaffine manifold and $M$ be an $n$-dimensional manifold. Let $\bigwedge^{r} T \widetilde{M}$ (resp. $\bigwedge^{r} T^{*} \widetilde{M}$ ) be the exterior product bundle of degree $r$ of the tangent bundle $T \widetilde{M}$ (resp. the dual bundle $T^{*} \widetilde{M}$ ). Denote the bundle projections of $\bigwedge^{r} T \widetilde{M}$ and $\bigwedge^{r} T^{*} \widetilde{M}$ by the same symbol $\pi$. We define the set $C_{\wedge}(M, \widetilde{M})\left(\operatorname{resp} . C_{\wedge^{*}}(M, \widetilde{M})\right)$ by

$$
\begin{array}{r}
C_{\wedge}(M, \widetilde{M}):=\left\{F \in C^{\infty}\left(M, \bigwedge^{r} T \widetilde{M}\right) \mid \Pi(F(x)) \oplus(\pi \circ F)_{*}\left(T_{x} M\right)\right. \\
\left.=T_{(\pi \circ F)(x)} \widetilde{M}(x \in M)\right\} \\
\text { (resp. } C_{\wedge^{*}}(M, \widetilde{M}):=\left\{F \in C^{\infty}\left(M, \bigwedge^{r} T^{*} \widetilde{M}\right) \mid \operatorname{Ker} F(x)\right. \\
\left.\left.=(\pi \circ F)_{*}\left(T_{x} M\right)(x \in M)\right\}\right),
\end{array}
$$


where $\Pi$ is the natural projection of $\bigwedge^{r} T \widetilde{M}$ removed the zero section onto the Grassmann bundle $G_{r}(T \widetilde{M})$ of $r$-dimensional subspaces. Also, define a submersion $\Phi: C_{\wedge}(M, \widetilde{M}) \rightarrow C_{\wedge^{*}}(M, \widetilde{M})$ by $\Phi(F)(x)(F(x))=1$ and $\operatorname{Ker} \Phi(F)(x)=(\pi \circ F)_{*}\left(T_{x} M\right)(x \in M)$. For $F \in C_{\wedge}(M, \widetilde{M})$, we set $f:=$ $\pi \circ F, \nu:=\Phi(F)$ and $N_{x}:=\Pi(F(x))(x \in M)$. We call $\nu$ a transversal volume element map of $F$.

Remark 2.1 In the case where the ambient space $(\widetilde{M}, \widetilde{\nabla}, \widetilde{\theta})$ is an equiaffine space $\left(\mathbf{R}^{n+r}, \widetilde{\nabla}, \widetilde{\omega}\right)$, the tansversal volume element map $\nu$ coincides with a transversal volume element map (of $F$ ) defined in $[\mathrm{KT}]$ under the identification of $\bigwedge^{r} T_{f(x)}^{*} \mathbf{R}^{n+r}(x \in M)$ with $\bigwedge^{r}\left(\mathbf{R}^{n+r}\right)^{*}$ (see Fig. 2).

Denote by $\operatorname{pr}_{T}\left(\right.$ resp. $\left.\operatorname{pr}_{N}\right)$ the projection of $f^{*} T \widetilde{M}$ onto $f_{*} T M$ (resp. $\left.N\right)$. We define a connection $\nabla$ on $M$ and a $N$-valued symmetric $(0,2)$-tensor field $h$ on $M$ by $f_{*}\left(\nabla_{X} Y\right)=\operatorname{pr}_{T}\left(\widetilde{\nabla}_{X} f_{*} Y\right)$ and $h(X, Y):=\operatorname{pr}_{N}\left(\widetilde{\nabla}_{X} f_{*} Y\right)$ $(X, Y \in T M)$, respectively. The quantities $\nabla$ (resp. $h$ ) is called the connection induced from $\widetilde{\nabla}$ by $F$ (resp. the affine fundamental form of $F$ ). Then we call $F$ an affine immersion of $(M, \nabla)$ into $(\widetilde{M}, \widetilde{\nabla})$. Also, we define a section $A$ of the tensor bundle $N^{*} \otimes T^{*} M \otimes T M$ and a connection $\nabla^{\perp}$ of $N$ by $f_{*}\left(A_{\xi} X\right)=-\operatorname{pr}_{T}\left(\widetilde{\nabla}_{X} \xi\right)$ and $\nabla_{X}^{\perp} \xi=\operatorname{pr}_{N}\left(\widetilde{\nabla}_{X} \xi\right)(X \in T M, \xi \in N)$, respectively. The quantities $A$ (resp. $\nabla^{\perp}$ ) is called the affine shape tensor (resp. the transversal connection) of $F$. Define a section $H$ of $N^{*}$ by $H(\xi)=(1 / n) \operatorname{tr} A_{\xi}(\xi \in N)$. The section $H$ is called the affine mean curvature function of $F$. Set $\theta^{\perp}:=\left.\nu\right|_{N \times \cdots \times N}$, which is called the transversal volume element of $F$. Define a volume element $\theta$ on $M$ by

$$
\theta\left(X_{1}, \ldots, X_{n}\right)=\frac{\widetilde{\theta}\left(f_{*} X_{1}, \ldots, f_{*} X_{n}, \xi_{1}, \ldots, \xi_{r}\right)}{\theta^{\perp}\left(\xi_{1}, \ldots, \xi_{r}\right)} \quad\left(X_{1}, \ldots, X_{n} \in T M\right),
$$

where $\left(\xi_{1}, \ldots, \xi_{r}\right)$ is an arbitrary frame of $N$. This volume element $\theta$ is called the induced volume element of $F$. Define a section $\tau$ of $f^{*} \wedge^{n} T^{*} \widetilde{M}$ by $\tau \circ f_{*}^{n}=\theta$ and $\operatorname{Ker} \tau=N$, where $f_{*}^{n}:=f_{*} \times \cdots \times f_{*}$ (n-times). We call $\tau$ a tangential volume element map of $F$. If $\nabla \theta=0$, then we call $F$ an equiaffine immersion of $(M, \nabla, \theta)$ into $(\widetilde{M}, \widetilde{\nabla}, \widetilde{\theta})$.

Remark 2.2 Let $\left(f, N, \theta^{\perp}\right)$ be an equiaffine immersion of an equiaffine manifold $(M, \nabla, \theta)$ into another equiaffine manifold $(\widetilde{M}, \widetilde{\nabla}, \widetilde{\theta})$ in the sense of $[\mathrm{K}]$, where $N$ is the transversal bundle and $\theta^{\perp}$ is the transversal volume element (hence $\left.\theta^{\perp} \in \Gamma\left(\bigwedge^{r} N^{*}\right)(r:=\operatorname{dim} \widetilde{M}-\operatorname{dim} M)\right)$. Then an 


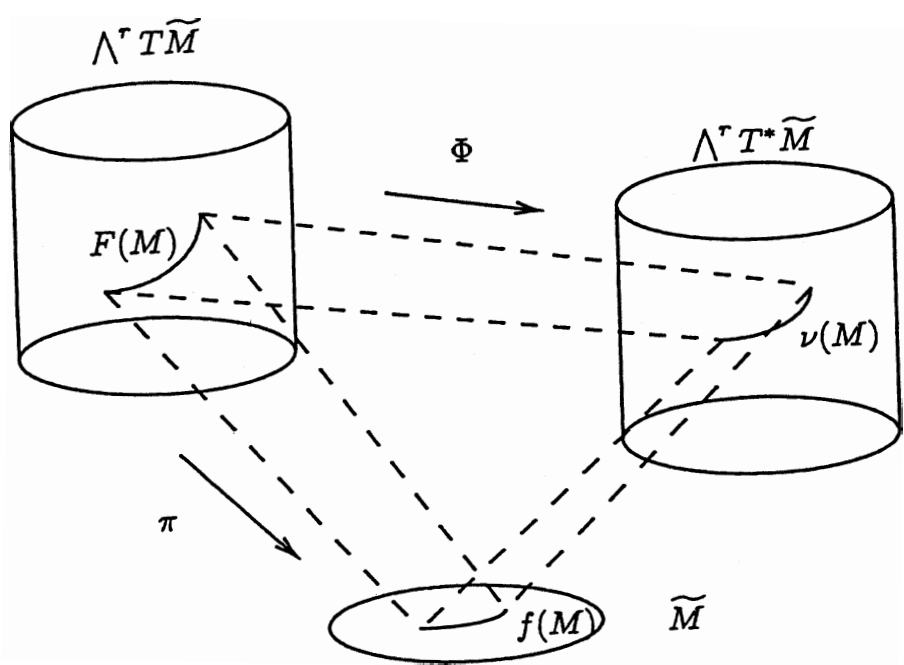

Fig. 1.

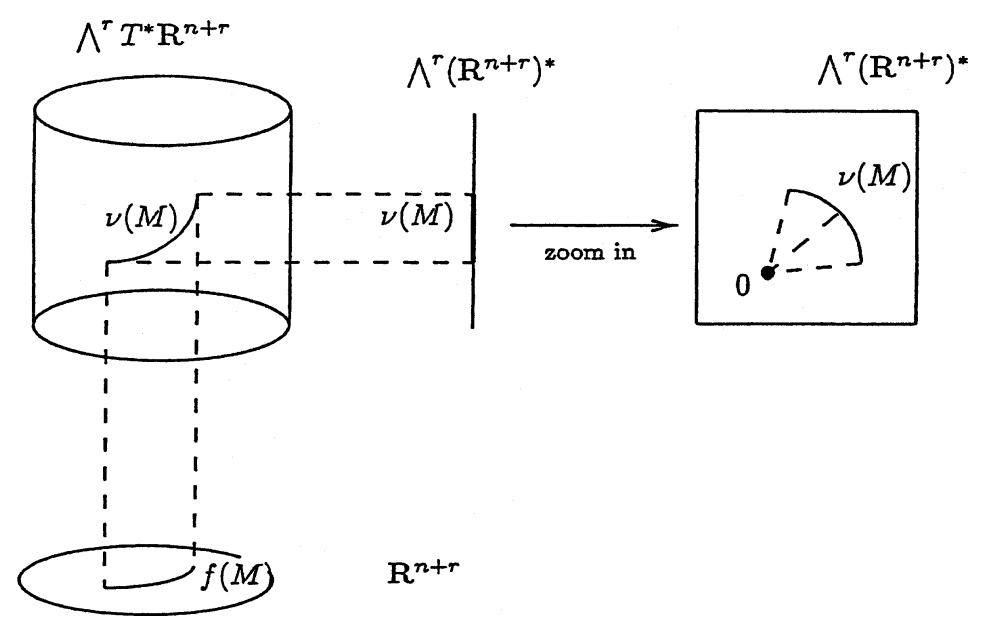

Fig. 2.

immersion $F: M \hookrightarrow \bigwedge^{r} T \widetilde{M}$ is defined by $F(x):=\xi_{1}^{x} \wedge \cdots \wedge \xi_{r}^{x}(x \in M)$, where $\left(\xi_{1}^{x}, \ldots, \xi_{r}^{x}\right)$ is a frame of $N_{x}$ with $\theta_{x}^{\perp}\left(\xi_{1}^{x}, \ldots, \xi_{r}^{x}\right)=1$. This map $F$ is an equiaffine immersion of $(M, \nabla, \theta)$ into $(\widetilde{M}, \widetilde{\nabla}, \widetilde{\theta})$ in the above sense. In particular, if $(f, \xi):(M, \nabla, \theta) \hookrightarrow(\widetilde{M}, \widetilde{\nabla}, \widetilde{\theta})$ is an equiaffine immersion of codimension one ( $\xi$ : the equiaffine transversal vector field), then an immersion $F: M \hookrightarrow T \widetilde{M}$ defined by $F(x):=\xi_{x}(x \in M)$ is an equiaffine 
immersion in the above sense.

Let $E(M,(\widetilde{M}, \widetilde{\nabla}, \widetilde{\theta})):=\left\{F \in C_{\wedge}(M, \widetilde{M}) \mid F\right.$ : equiaffine (i.e., $\left.\left.\nabla \theta=0\right)\right\}$. For simplicity, we denote this set by $E(M)$. Let $F \in E(M)$. Take a frame $\left(\xi_{1}, \ldots, \xi_{r}\right)$ of $N$ with $\theta^{\perp}\left(\xi_{1}, \ldots, \xi_{r}\right)=1$ and let $h=h^{\rho} \otimes \xi_{\rho}$, where $\rho$ runs $\{1, \ldots, r\}$ and we use the Einstein's rule. In the sequel, we use this rule. Define a $(0,2 r)$-tensor field $\widetilde{h}$ on $M$ by

$$
\begin{aligned}
& \widetilde{h}\left(X_{1}, X_{2}, \ldots, X_{2 r-1}, X_{2 r}\right) \\
& \quad:=\delta_{\rho_{1} \cdots \rho_{r}} h^{\rho_{1}}\left(X_{1}, X_{2}\right) \cdots h^{\rho_{r}}\left(X_{2 r-1}, X_{2 r}\right)
\end{aligned}
$$

for $X_{1}, \ldots, X_{2 r} \in T M$, where $\delta_{\rho_{1} \ldots \rho_{r}}$ is given by

$$
\delta_{\rho_{1} \cdots \rho_{r}}:=\left\{\begin{array}{clc}
\operatorname{sgn}\left(\begin{array}{ccc}
1 & \cdots & r \\
\rho_{1} & \cdots & \rho_{r}
\end{array}\right) & \left(\left\{\rho_{1}, \ldots, \rho_{r}\right\}=\{1, \ldots, r\}\right) \\
0 & \left(\left\{\rho_{1}, \ldots, \rho_{r}\right\} \neq\{1, \ldots, r\}\right) .
\end{array}\right.
$$

It is easy to show that $\widetilde{h}$ is well-defined, that is, it is independent of the choice of a frame $\left(\xi_{1}, \ldots, \xi_{r}\right)$ of $N$ with $\theta^{\perp}\left(\xi_{1}, \ldots, \xi_{r}\right)=1$. An $n$-form $\omega_{h}$ on $M$ is defined by

$$
\begin{aligned}
\omega_{h}\left(\partial_{1}, \ldots, \partial_{n}\right) & =\left|\frac{1}{n !} \delta^{i_{1}^{1} \cdots i_{1}^{n}} \cdots \delta^{i_{2 r}^{1} \cdots i_{2 r}^{n}} \widetilde{h}_{i_{1}^{1} \cdots i_{2 r}^{1}} \cdots \widetilde{h}_{i_{1}^{n} \cdots i_{2 r}^{n}}\right|^{1 /(2 r)} \\
( & \left.=\left|\delta^{i_{2}^{1} \cdots i_{2}^{n}} \cdots \delta^{i_{2 r}^{1} \cdots i_{2 r}^{n}} \widetilde{h}_{1 i_{2}^{1} \cdots i_{2 r}^{1}} \cdots \widetilde{h}_{n i_{2}^{n} \cdots i_{2 r}^{n}}\right|^{1 /(2 r)}\right),
\end{aligned}
$$

where $\left(\partial_{1}, \ldots, \partial_{n}\right)$ is the natural base of a local coordinate $\left(x^{1}, \ldots, x^{n}\right)$ of $M$ which is positive with respect to $\theta . \widetilde{h}_{i_{1}^{j} \cdots i_{2 r}^{j}}(j=1, \ldots, n)$ are components of $\widetilde{h}$ with respect to $\left(x^{1}, \ldots, x^{n}\right)$ and

$$
\delta_{j_{j}^{1} \cdots i_{j}^{n}}:=\left\{\begin{array}{ccc}
\operatorname{sgn}\left(\begin{array}{ccc}
1 & \cdots & n \\
i_{j}^{1} & \cdots & i_{j}^{n}
\end{array}\right) & \left(\left\{i_{j}^{1}, \ldots, i_{j}^{n}\right\}=\{1, \ldots, n\}\right) \\
0 & \left(\left\{i_{j}^{1}, \ldots, i_{j}^{n}\right\} \neq\{1, \ldots, n\}\right) .
\end{array}\right.
$$

It is easy to show that $\omega_{h}$ is well-defined, that is, it is independent of the choice of a local coordinate $\left(x^{1}, \ldots, x^{n}\right)$ of $M$ which is positive with respect to $\theta$. If $\omega_{h}$ is a volume element on $M$, that is, it does not vanish at each point of $M$, then we call $F$ a non-degenerate equiaffine immersion. Denote by $E_{N D}(M)$ the set of all non-degenerate equiaffine immersions of $M$ into $(\widetilde{M}, \widetilde{\nabla}, \widetilde{\theta})$. Let $f$ be an immersion of $M$ into $(\widetilde{M}, \widetilde{\nabla}, \widetilde{\theta})$. If there exists $F \in$ 
$E_{N D}(M)$ with $\pi \circ F=f$, then $f$ is called a non-degenerate immersion. Then, for any $F \in E(M)$ with $\pi \circ F=f$, we have $F \in E_{N D}(M)$. Denote by $N D(M)$ the set of all non-degenerate immersions of $M$ into $(\widetilde{M}, \widetilde{\nabla}, \widetilde{\theta})$. Define a $(2 r, 0)$-tensor field $\widetilde{h}^{-1}$ on $M$ by

$$
\begin{aligned}
\widetilde{h}^{-1}:= & \frac{1}{(n-1) ! \omega_{h}\left(\partial_{1}, \ldots, \partial_{n}\right)^{2 r}} \delta^{i_{1} j_{1}^{1} \cdots j_{1}^{n-1}} \cdots \delta^{i_{2 r} j_{2 r}^{1} \cdots j_{2 r}^{n-1}} \\
& \times \widetilde{h}_{j_{1}^{1} \cdots j_{2 r}^{1}} \cdots \widetilde{h}_{j_{1}^{n-1} \ldots j_{2 r}^{n-1}} \partial_{i_{1}} \otimes \cdots \otimes \partial_{i_{2 r}},
\end{aligned}
$$

where $\widetilde{h}_{j_{1}^{k} \ldots j_{2 r}^{k}}(k=1, \ldots, n-1)$ are the component of $\widetilde{h}$ with respect to $\left(x^{1}, \ldots, x^{n}\right)$. It is easy to show that $\widetilde{h}^{-1}$ is well-defined. Denote by $\widetilde{h}^{i_{1} \cdots i_{2 r}}$ the component of $\widetilde{h}^{-1}$ with respect to a local coordinate $\left(x^{1}, \ldots, x^{n}\right)$. This tensor field $\widetilde{h}^{-1}$ is called the pseudo-inverse of $\widetilde{h}$. Also, define a section $\mathfrak{h}$ of the tensor bundle $N^{*} \otimes T M \otimes T M$ by

$$
\mathfrak{h}\left(\xi_{\rho}\right)=r \delta_{\rho \rho_{2} \cdots \rho_{r}} h_{k_{3} k_{4}}^{\rho_{2}} \cdots h_{k_{2 r-1} k_{2 r}}^{\rho_{r}} \widetilde{h}^{i j k_{3} k_{4} \cdots k_{2 r-1} k_{2 r}} \partial_{i} \otimes \partial_{j},
$$

where $h_{k l}^{\rho}$ is defined by $h\left(\partial_{k}, \partial_{l}\right)=h_{k l}^{\rho} \xi_{\rho}$. It is easy to show that $\mathfrak{h}$ is welldefined. This section $\mathfrak{h}$ is called the pseudo-inverse of $h$. Denote by $\mathfrak{h}_{\rho}^{i j}$ the component of $\mathfrak{h}$ with respect to $\left(\partial_{1}, \ldots, \partial_{n}\right)$ and $\left(\xi_{1}, \ldots, \xi_{r}\right)$. We prepare the following lemma.

Lemma 2.1 For components $h_{i j}^{\rho}\left(\right.$ resp. $\left.\mathfrak{h}_{\rho}^{i j}\right)$ of $h$ (resp. h) with respect to $\left(\partial_{1}, \ldots, \partial_{n}\right)$ and $\left(\xi_{1}, \ldots, \xi_{r}\right)$, we have $h_{i j}^{\rho} \mathfrak{h}_{\gamma}^{i j}=n \delta_{\gamma}^{\rho}$ and $h_{i j}^{\rho} \mathfrak{h}_{\rho}^{i k}=r \delta_{j}^{k}$.

See Lemma 4.13 of [Wi] about the proof of this lemma. Denote by $\hat{\nabla}$ the connection of $N^{*} \otimes T M \otimes T M$ induced from $\nabla$ and $\nabla^{\perp}$. Define a section $T$ of $N^{*} \otimes T M$ by $T_{\rho}^{i}=\left(\widehat{\nabla}_{j} \mathfrak{h}\right)_{\rho}^{j i}$. For each section $\xi$ of $N$, the tangent vector field $T(\xi)$ of $M$ is called the Tchebychev vector field of $F$. We call $T$ the Tchebychev tensor field of $F$. Define a 1 -form $\widehat{T}$ on $M$ by $\widehat{T}\left(\partial_{j}\right)=T_{\rho}^{i} h_{i j}^{\rho}$. We call $\widehat{T}$ the Tchebychev form of $F$. Also, we define a section $T^{e}$ of $f^{*} T^{*} \widetilde{M} \otimes T M$ by $\left.T^{e}\right|_{N}=T$ and $\left.T^{e}\right|_{f_{*} T M}=0$. We call $T^{e}$ the extended Tchebychev tensor field of $F$.

Proposition 2.2 For $F \in E_{N D}(M)$, we have $\widehat{T}=2 r d \log \frac{\omega_{h}}{\theta}$. In particular, the form $\widehat{T}$ is exact.

Proof. This relation directly follows from (5.2) of [Wi] and the equiaffinity of $F$. 


\section{The variational formulas for the volume function over the space of all equiaffine immersions}

Let $E(M)$ be the space of all equiaffine immersions of a manifold $M$ into an equiaffine manifold $(\widetilde{M}, \widetilde{\nabla}, \widetilde{\theta})$. Let $\pi$ be the bundle projection of $\bigwedge^{r}(T \widetilde{M})$. For an immersion $\phi$ of $M \backslash D(D$ : a relatively compact domain with smooth boundary of $M)$ into $\widetilde{M}$, we set $E(M)_{\phi}:=\{F \in E(M) \mid \pi \circ$ $F=\phi$ on $M \backslash D\}$ and $\left.E(M)_{\phi}\right|_{D}:=\left\{\left.F\right|_{D} \mid F \in E(M)_{\phi}\right\}$. Define the volume $\operatorname{Vol}(F)$ of $\left.F \in E(M)_{\phi}\right|_{D}$ by $\operatorname{Vol}(F)=\int_{D} \theta$, where $\theta$ is the induced volume element of $F$. Let $K$ be the connection map of the connection (which is also denote by $\widetilde{\nabla}$ ) on $\bigwedge^{r} T^{*} \widetilde{M}$ induced from $\widetilde{\nabla}$, that is, the element of $\Gamma\left(T^{*}\left(\bigwedge^{r} T^{*} \widetilde{M}\right) \otimes V\right)$ defined by

$$
\left(K\left(\left.\frac{d \mu}{d t}\right|_{t=0}\right)=\widetilde{\nabla}_{\pi_{*}\left(\left.\frac{d \mu}{d t}\right|_{t=0}\right)} \mu\right.
$$

for each curve $\mu:(-\varepsilon, \varepsilon) \rightarrow \bigwedge^{r} T^{*} \widetilde{M}$, where $V$ is the vertical distribution of $\bigwedge^{r} T^{*} \widetilde{M}, \pi$ is the bundle projection of $\bigwedge^{r} T^{*} \widetilde{M}$ and we identify $V_{\mu(0)}$ with the fibre $\bigwedge^{r} T_{\pi(\mu(0))}^{*} \widetilde{M}$. First we prove the following first and second variational formulas.

Theorem 3.1 Let $\operatorname{dim} M=n, \operatorname{dim} \widetilde{M}=n+r, D$ be a relatively compact domain with smooth boundary of $M$ and $\phi$ be an immersion of $M \backslash D$ into $\widetilde{M}$. Let $\left.F \in E(M)_{\phi}\right|_{D}, F_{t}(-\varepsilon<t<\varepsilon)$ be a variation of $F$ in $\left.E(M)_{\phi}\right|_{D}$ and $\nu_{t}$ be the transversal volume element map of $F_{t}$. Let $v:=\left.\frac{d F_{t}}{d t}\right|_{t=0}$ and $v_{\nu}:=$ $\left.\frac{d \nu_{t}}{d t}\right|_{t=0}$.

(i) We have the following first variational formula:

$$
\left.\frac{d}{d t}\right|_{t=0} \operatorname{Vol}\left(F_{t}\right)=-\int_{D}\left(n H\left(\left(\pi_{*} v\right)_{N}\right)+K\left(v_{\nu}\right)\left(\theta^{\perp-1}(1)\right)\right) \theta,
$$

where $H$ is the affine mean curvature function of $F, \pi$ is the bundle projection of $\bigwedge^{r}(T \widetilde{M}),\left(\pi_{*} v\right)_{N}$ is the $N$-component of $\pi_{*} v(N$ : the transversal bundle of $F$ ) and $K\left(v_{\nu}\right)\left(\theta^{\perp-1}(1)\right)$ implies $K\left(v_{\nu}\right)\left(\xi_{1}, \ldots, \xi_{r}\right)$ as $\theta^{\perp}\left(\xi_{1}, \ldots, \xi_{r}\right)=1$. 
(ii) We have the following second variational formula:

$$
\begin{aligned}
&\left.\frac{d^{2}}{d t^{2}}\right|_{t=0} \operatorname{Vol}\left(F_{t}\right) \\
&=\int_{D}\left(\left(n H\left(\left(\pi_{*} v\right)_{N}\right)+K\left(v_{\nu}\right)\left(\theta^{\perp-1}(1)\right)\right)^{2}\right. \\
& \quad-2 \operatorname{div}_{\nabla}\left(\left(\pi_{*} v\right)_{T}\right)\left(n H\left(\left(\pi_{*} v\right)_{N}\right)+K\left(v_{\nu}\right)\left(\theta^{\perp-1}(1)\right)\right) \\
& \quad-\operatorname{tr}_{T} \widetilde{R}\left(\cdot, \pi_{*} v\right) \pi_{*} v-\operatorname{tr}\left(\left(A_{\left(\pi_{*} v\right)_{N}}-\nabla\left(\pi_{*} v\right)_{T}\right)^{2}\right) \\
& \quad+\left(\operatorname{div}_{\nabla}\left(\left(\pi_{*} v\right)_{T}\right)\right)^{2}+K\left(v_{\nu}\right)\left(\theta^{\perp-1}(1)\right)^{2} \\
&\left.-K\left(\left.\frac{d}{d t}\right|_{t=0} K\left(\frac{d \nu_{t}}{d t}\right)\right)\left(\theta^{\perp-1}(1)\right)-n H\left(\left(\widetilde{\nabla}_{\left.\partial_{t}\right|_{t=0}} \delta_{*} \partial_{t}\right)_{N}\right)\right) \theta
\end{aligned}
$$

Here $\widetilde{R}$ is the curvature tensor of $\widetilde{\nabla}, \operatorname{tr}_{T} \widetilde{R}\left(\cdot, \pi_{*} v\right) \pi_{*} v$ is the trace of $\left.\operatorname{pr}_{T} \circ \widetilde{R}\left(\cdot, \pi_{*} v\right) \pi_{*} v\right|_{f_{*} T M}(f:=\pi \circ F), A$ (resp. $\left.\nabla\right)$ is the affine shape tensor (resp. the induced connection) of $F,\left(\pi_{*} v\right)_{T}:=\operatorname{pr}_{T}\left(\pi_{*} v\right)$ and $\delta$ is a map of $D \times(-\varepsilon, \varepsilon)$ into $\widetilde{M}$ defined by $\delta(x, t):=\left(\pi \circ F_{t}\right)(x)$, where $\operatorname{pr}_{T}$ is the projection of $f^{*} T \widetilde{M}$ onto $f_{*} T M$ with respect to the decomposition $f^{*} T \widetilde{M}=$ $f_{*} T M \oplus N$.

Proof. Let $N_{t}, \theta_{t}, \theta_{t}^{\perp}, h_{t}, A^{t}, \nabla^{t}$ and $\nabla^{\perp_{t}}$ be the quantities for $F_{t}$. Take a local coordinate $\left(U,\left(x^{1}, \ldots, x^{n}\right)\right)$ of $M$ with $U \subset D$. For simplicity, set $\partial_{i}:=\partial / \partial x^{i}(i=1, \ldots, n)$. Let $\left(\xi_{1}^{t}, \ldots, \xi_{r}^{t}\right)$ be a local frame field of $N_{t}$ on $U$ with $\theta_{t}^{\perp}\left(\xi_{1}^{t}, \ldots, \xi_{r}^{t}\right)=1$. Set $\delta(t, x):=f_{t}(x)(x \in M)$. Denote by the same symbol $\widetilde{\nabla}$ the connection of $\delta^{*} T \widetilde{M}$ induced from $\widetilde{\nabla}$ by $\delta$. Let $\left(\widetilde{\nabla}_{\partial_{t}} \xi_{\rho}^{t}\right)_{N_{t}}=$ $\widetilde{\tau}_{\rho}^{\mu} \xi_{\mu}^{t}$. Then we have

$$
\begin{aligned}
\left.\frac{d}{d t}\right|_{t=0} \theta_{t}\left(\partial_{1}, \ldots, \partial_{n}\right) & =\left.\frac{d}{d t}\right|_{t=0} \widetilde{\theta}\left(f_{t *} \partial_{1}, \ldots, f_{t *} \partial_{n}, \xi_{1}^{t}, \ldots, \xi_{r}^{t}\right) \\
= & \sum_{i=1}^{n} \tilde{\theta}\left(f_{*} \partial_{1}, \ldots,\left.\left(\widetilde{\nabla}_{\partial_{i}} \delta_{*} \partial_{t}\right)\right|_{t=0}, \ldots, f_{*} \partial_{n}, \xi_{1}^{0}, \ldots, \xi_{r}^{0}\right) \\
& +\widetilde{\tau}_{\rho}^{\rho} \theta\left(\partial_{1}, \ldots, \partial_{n}\right) .
\end{aligned}
$$

Also, we have

$$
\left(\left.\left(\widetilde{\nabla}_{\partial_{i}} \delta_{*} \partial_{t}\right)\right|_{t=0}\right)_{T}=\left(\widetilde{\nabla}_{\partial_{i}} \pi_{*} v\right)_{T}=-A_{\left(\pi_{*} v\right)_{N}} \partial_{i}+\nabla_{\partial_{i}}\left(\pi_{*} v\right)_{T},
$$




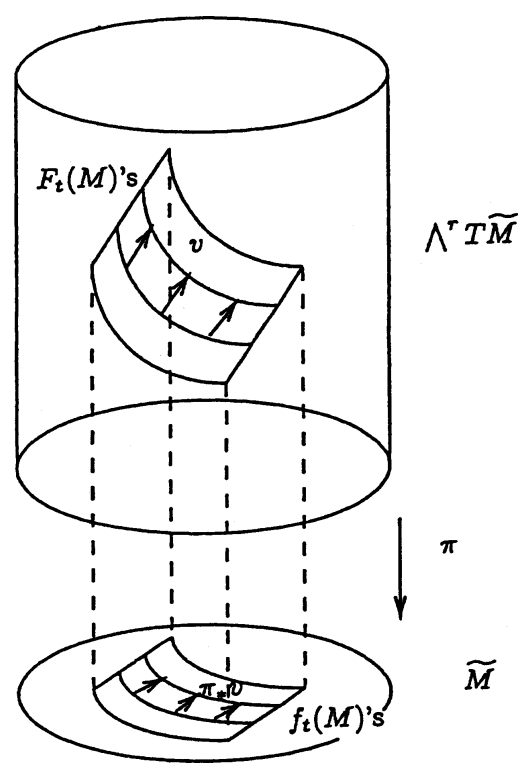

Fig. 3.

where $(\cdot)_{T}$ implies the element of $T M$ such that $f_{*}\left((\cdot)_{T}\right)$ is equal to the $f_{*} T M$-component of $(\cdot)$. From (3.1) and (3.2), we have

$$
\begin{aligned}
& \left.\frac{d}{d t}\right|_{t=0} \theta_{t}\left(\partial_{1}, \ldots, \partial_{n}\right) \\
& \quad=\sum_{i=1}^{n} \theta\left(\partial_{1}, \ldots,-A_{\left(\pi_{*} v\right)_{N}} \partial_{i}+\nabla_{\partial_{i}}\left(\pi_{*} v\right)_{T}, \ldots, \partial_{n}\right)+\widetilde{\tau}_{\rho}^{\rho} \theta\left(\partial_{1}, \ldots, \partial_{n}\right) \\
& \quad=\left(-n H\left(\left(\pi_{*} v\right)_{N}\right)+\operatorname{div}_{\nabla}\left(\pi_{*} v\right)_{T}+\widetilde{\tau}_{\rho}^{\rho}\right) \theta\left(\partial_{1}, \ldots, \partial_{n}\right),
\end{aligned}
$$

where $\operatorname{div}_{\nabla}\left(\pi_{*} v\right)_{T}$ is the divergence of $\left(\pi_{*} v\right)_{T}$ with respect to $\nabla$. Also, we have

$$
\begin{aligned}
\left.\partial_{t}\right|_{t=0} \theta_{t}^{\perp}\left(\xi_{1}^{t}, \ldots, \xi_{r}^{t}\right) & =\left.\partial_{t}\right|_{t=0} \nu_{t}\left(\xi_{1}^{t}, \ldots, \xi_{r}^{t}\right) \\
& =K\left(v_{\nu}\right)\left(\theta^{\perp-1}(1)\right)+\widetilde{\tau}_{\rho}^{\rho},
\end{aligned}
$$

which vanishes because of $\theta_{t}^{\perp}\left(\xi_{1}^{t}, \ldots, \xi_{r}^{t}\right)=1$. That is, we have $\widetilde{\tau}_{\rho}^{\rho}=$ $-K\left(v_{\nu}\right)\left(\theta^{\perp-1}(1)\right)$. From $\nabla \theta=0$ and $\left.\left(\pi_{*} v\right)_{T}\right|_{\partial D}=0$, it follows that $\int_{D} \operatorname{div}_{\nabla}\left(\pi_{*} v\right)_{T} \theta=0$ (see Lemma 6.1 and (2.11) of [Wi]). By integrating (3.3) and using these relations, we can obtain the desired first variational 
formula in the statement (i).

Next we shall show the second variational formula in (ii). We have

$$
\begin{aligned}
& \left.\frac{d^{2}}{d t^{2}}\right|_{t=0} \theta_{t}\left(\partial_{1}, \ldots, \partial_{n}\right) \\
& =\left.\frac{d^{2}}{d t^{2}}\right|_{t=0} \widetilde{\theta}\left(f_{t *} \partial_{1}, \ldots, f_{t *} \partial_{n}, \xi_{1}^{t}, \ldots, \xi_{r}^{t}\right) \\
& =2 \sum_{1 \leq i<j \leq n} \widetilde{\theta}\left(f_{*} \partial_{1}, \ldots,\left.\widetilde{\nabla}_{\partial_{t}} \delta_{*} \partial_{i}\right|_{t=0}, \ldots,\left.\widetilde{\nabla}_{\partial_{t}} \delta_{*} \partial_{j}\right|_{t=0}, \ldots, f_{*} \partial_{n}, \xi_{1}^{0}, \ldots, \xi_{r}^{0}\right) \\
& +\sum_{i=1}^{n} \widetilde{\theta}\left(f_{*} \partial_{1}, \ldots,\left.\widetilde{\nabla}_{\partial_{t}} \widetilde{\nabla}_{\partial_{t}} \delta_{*} \partial_{i}\right|_{t=0}, \ldots, f_{*} \partial_{n}, \xi_{1}^{0}, \ldots, \xi_{r}^{0}\right) \\
& +2 \sum_{1 \leq \rho<\mu \leq r} \widetilde{\theta}\left(f_{*} \partial_{1}, \ldots, f_{*} \partial_{n}, \xi_{1}^{0}, \ldots,\left.\widetilde{\nabla}_{\partial_{t}} \xi_{\rho}^{t}\right|_{t=0}, \ldots,\left.\widetilde{\nabla}_{\partial_{t}} \xi_{\mu}^{t}\right|_{t=0}, \ldots, \xi_{r}^{0}\right) \\
& +\sum_{\rho=1}^{r} \widetilde{\theta}\left(f_{*} \partial_{1}, \ldots, f_{*} \partial_{n}, \xi_{1}^{0}, \ldots,\left.\widetilde{\nabla}_{\partial_{t}} \widetilde{\nabla}_{\partial_{t}} \xi_{\rho}^{t}\right|_{t=0}, \ldots, \xi_{r}^{0}\right) \\
& +2 \sum_{i=1}^{n} \sum_{\rho=1}^{r} \widetilde{\theta}\left(f_{*} \partial_{1}, \ldots,\left.\widetilde{\nabla}_{\partial_{t}} \delta_{*} \partial_{i}\right|_{t=0}, \ldots, f_{*} \partial_{n}, \xi_{1}^{0}, \ldots,\left.\widetilde{\nabla}_{\partial_{t}} \xi_{\rho}^{t}\right|_{t=0}, \ldots, \xi_{r}^{0}\right) .
\end{aligned}
$$

The first term $\left(*_{1}\right)$ in the right-hand side of $(3.4)$ is rewritten as follows:

$$
\begin{aligned}
\left(*_{1}\right)= & \left\{n^{2} H\left(\left(\pi_{*} v\right)_{N}\right)^{2}-\operatorname{tr}\left(\left(A_{\left(\pi_{*} v\right)_{N}}\right)^{2}\right)-2 n H\left(\left(\pi_{*} v\right)_{N}\right) \operatorname{tr} \nabla\left(\pi_{*} v\right)_{T}\right. \\
& +2 \operatorname{tr}\left(A_{\left(\pi_{*} v\right)_{N}} \circ \nabla\left(\pi_{*} v\right)_{T}\right)+\left(\operatorname{tr} \nabla\left(\pi_{*} v\right)_{T}\right)^{2} \\
& \left.-\operatorname{tr}\left(\left(\nabla\left(\pi_{*} v\right)_{T}\right)^{2}\right)\right\} \theta\left(\partial_{1}, \ldots, \partial_{n}\right) .
\end{aligned}
$$

The second term $\left(*_{2}\right)$ in the right-hand side of (3.4) is rewritten as follows:

$$
\begin{aligned}
\left(*_{2}\right)= & \sum_{i=1}^{n} \theta\left(\partial_{1}, \ldots,\left(\widetilde{\nabla}_{\partial_{t} \mid t=0} \widetilde{\nabla}_{\partial_{i}} \delta_{*} \partial_{t}\right)_{T}, \ldots, \partial_{n}\right) \\
= & \sum_{i=1}^{n} \theta\left(\partial_{1}, \ldots,-\left(\widetilde{R}\left(f_{*} \partial_{i}, \pi_{*} v\right) \pi_{*} v\right)_{T}+\left(\widetilde{\nabla}_{\partial_{i}} \widetilde{\nabla}_{\left.\partial_{t}\right|_{t=0}} \delta_{*} \partial_{t}\right)_{T}, \ldots, \partial_{n}\right) \\
= & -\left(\operatorname{tr}_{T} \widetilde{R}\left(\cdot, \pi_{*} v\right) \pi_{*} v\right) \theta\left(\partial_{1}, \ldots, \partial_{n}\right) \\
& +\sum_{i=1}^{n} \theta\left(\partial_{1}, \ldots,-A_{\left(\widetilde{\nabla}_{\left.\partial_{t}\right|_{t=0}} \delta_{*} \partial_{t}\right)_{N}} \partial_{i}+\nabla_{\partial_{i}}\left(\widetilde{\nabla}_{\left.\partial_{t}\right|_{t=0}} \delta_{*} \partial_{t}\right)_{T}, \ldots, \partial_{n}\right)
\end{aligned}
$$




$$
\begin{aligned}
= & \left(-\operatorname{tr}_{T} \widetilde{R}\left(\cdot, \pi_{*} v\right) \pi_{*} v-n H\left(\left(\widetilde{\nabla}_{\left.\partial_{t}\right|_{t=0}} \delta_{*} \partial_{t}\right)_{N}\right)\right. \\
& \left.+\operatorname{div}_{\nabla}\left(\widetilde{\nabla}_{\left.\partial_{t}\right|_{t=0}} \delta_{*} \partial_{t}\right)_{T}\right) \theta\left(\partial_{1}, \ldots, \partial_{n}\right) .
\end{aligned}
$$

The sum $\left(*_{34}\right)$ of the third term and the fourth one in the right-hand side of (3.4) is rewritten as follows:

$$
\begin{aligned}
\left(*_{34}\right)= & \left(2 \sum_{1 \leq \rho<\mu \leq r} \nu\left(\xi_{1}^{0}, \ldots,\left.\widetilde{\nabla}_{\partial_{t}} \xi_{\rho}^{t}\right|_{t=0}, \ldots,\left.\widetilde{\nabla}_{\partial_{t}} \xi_{\mu}^{t}\right|_{t=0}, \ldots, \xi_{r}^{0}\right)\right. \\
& \left.+\sum_{\rho=1}^{r} \nu\left(\xi_{1}^{0}, \ldots,\left.\widetilde{\nabla}_{\partial_{t}} \widetilde{\nabla}_{\partial_{t}} \xi_{\rho}^{t}\right|_{t=0}, \ldots, \xi_{r}^{0}\right)\right) \theta\left(\partial_{1}, \ldots, \partial_{n}\right) \\
= & \left(-\left(\widetilde{\nabla}_{\left.\partial_{t}\right|_{t=0}}\left(\widetilde{\nabla}_{\partial_{t}} \nu_{t}\right)\right)\left(\xi_{1}, \ldots, \xi_{r}\right)\right. \\
& \left.-2 \sum_{\rho=1}^{r}\left(\widetilde{\nabla}_{\left.\partial_{t}\right|_{t=0}} \nu_{t}\right)\left(\xi_{1}, \ldots, \widetilde{\nabla}_{\left.\partial_{t}\right|_{t=0}} \xi_{\rho}^{t}, \ldots, \xi_{r}\right)\right) \theta\left(\partial_{1}, \ldots, \partial_{n}\right) \\
= & \left(-K\left(\left.\frac{d}{d t}\right|_{t=0} K\left(\frac{d \nu_{t}}{d t}\right)\right)\left(\theta^{\perp-1}(1)\right)+2 K\left(v_{\nu}\right)\left(\theta^{\perp-1}(1)\right)^{2}\right) \\
& \theta\left(\partial_{1}, \ldots, \partial_{n}\right),
\end{aligned}
$$

where we use $\left.\frac{d^{2}}{d t^{2}}\right|_{t=0} \nu_{t}\left(\xi_{1}^{t}, \ldots, \xi_{r}^{t}\right)=0$. The fifth term $\left(*_{5}\right)$ in the right-hand side of (3.4) is rewritten as follows:

$$
\begin{gathered}
\left(*_{5}\right)=2 K\left(v_{\nu}\right)\left(\theta^{\perp-1}(1)\right)\left(n H\left(\left(\pi_{*} v\right)_{N}\right)-\operatorname{div}_{\nabla}\left(\pi_{*} v\right)_{T}\right) \\
\theta\left(\partial_{1}, \ldots, \partial_{n}\right) .
\end{gathered}
$$

These relations $(3.4) \sim(3.8)$ together with the arbitrariness of $\left(U,\left(x^{1}, \ldots, x^{n}\right)\right)$ deduces the desired second variational formula.

Remark 3.1 The relation (3.3) is equivalent to (6.5) of [Wi].

Now we state the following two facts.

Proposition 3.2 If $(\widetilde{\nabla}, \widetilde{\theta})$ is the equiaffine structure associated with a pseudo-Riemannian metric $\widetilde{g}$ of $\widetilde{M}$ and $F_{t}$ is a variation consisting of isometric immersions with respect to $\widetilde{g}$, then we have $K\left(v_{\nu}\right)\left(\theta^{\perp-1}(1)\right)=0$.

Proof. Let $\left(\xi_{1}^{t}, \ldots, \xi_{r}^{t}\right)$ be an orthonormal normal base of $f_{t}$ at $x$. Then we have $F_{t}(x)=\xi_{1}^{t} \wedge \cdots \wedge \xi_{r}^{t}$ and hence 


$$
K\left(v_{\nu}\right)\left(\theta^{\perp-1}(1)\right)=\widetilde{\tau}_{\rho}^{\rho}=\sum_{\rho=1}^{r} \widetilde{g}\left(\nabla_{\pi_{*} v}^{\perp} \xi_{\rho}^{t}, \xi_{\rho}^{0}\right)=0 .
$$

Proposition 3.3 The volume function over $\left.E(M)_{\phi}\right|_{D}$ has no critical point.

Proof. Take an arbitrary $\left.F \in E(M)_{\phi}\right|_{D}$. Define a variation $F_{t}$ of $F$ by $F_{t}(x)=(t+1) F(x)(x \in D)$. Since $F$ is equiaffine and the coefficient $(t+1)$ of the right-hand side is independent of the choice of $x \in D$, $F_{t}$ is also equiaffine. Hence we have $\left.F_{t} \in E(M)_{\phi}\right|_{D}$. Easily we can show $\left.\frac{d}{d t}\right|_{t=0} \operatorname{Vol}\left(F_{t}\right)>0$. Hence $F$ is not a critical point of the volume function over $E(M)$. This completes the proof.

When the variation consists of isometric immersions, the quantity $K\left(\left.\frac{d}{d t}\right|_{t=0} K\left(\frac{d \nu_{t}}{d t}\right)\right)\left(\theta^{\perp-1}(1)\right)$ in the second variational formula of (ii) of Theorem 3.1 is described as follows.

Proposition 3.4 If $(\widetilde{\nabla}, \widetilde{\theta})$ is the equiaffine structure associated with a pseudo-Riemannian metric $\widetilde{g}$ of $\widetilde{M}$ and $F_{t}$ is a variation consisting of isometric immersions, then we have

$$
K\left(\left.\frac{d}{d t}\right|_{t=0} K\left(\frac{d \nu_{t}}{d t}\right)\right)\left(\theta^{\perp-1}(1)\right)=-\left\|\nabla^{\perp}\left(\pi_{*} v\right)_{N}+h\left(\left(\pi_{*} v\right)_{T}, \cdot\right)\right\|_{\widetilde{g}}^{2}
$$

where $\|\cdot\|_{\tilde{g}}$ is the norm of $(\cdot)$ with respect to $\widetilde{g}$.

Proof. Define a map $\widetilde{\nu}$ of $M \times(-\varepsilon, \varepsilon)$ into $\bigwedge^{r} T^{*} \widetilde{M}$ by $\widetilde{\nu}(x, t)=\nu_{t}(x)$ $((x, t) \in M \times(-\varepsilon, \varepsilon))$, where $\nu_{t}$ is the transversal volume element map of $F_{t}$. Let $\left(\xi_{1}^{t}, \ldots, \xi_{r}^{t}\right)$ be an orthonormal frame field of $N_{t}$ and $\widetilde{\xi}_{i}(i=1, \ldots, r)$ be a section of $\delta^{*} T \widetilde{M}$ defined by $\widetilde{\xi}_{i}(x, t):=\left(\xi_{i}^{t}\right)_{x}((x, t) \in D \times(-\varepsilon, \varepsilon))$. Then we have

$$
\begin{aligned}
K( & \left.\left.\frac{d}{d t}\right|_{t=0} K\left(\frac{d \nu_{t}}{d t}\right)\right)\left(\theta^{\perp-1}(1)\right) \\
= & \left(\widetilde{\nabla}_{\left.\partial_{t}\right|_{t=0}}\left(\widetilde{\nabla}_{\partial_{t}} \widetilde{\nu}\right)\right)\left(\xi_{1}, \ldots, \xi_{r}\right) \\
= & \widetilde{\nabla}_{\left.\partial_{t}\right|_{t=0}}\left(\left(\widetilde{\nabla}_{\partial_{t}} \widetilde{\nu}\right)\left(\xi_{1}^{t}, \ldots, \xi_{r}^{t}\right)\right) \\
& \quad-\sum_{\rho=1}^{r}\left(\widetilde{\nabla}_{\left.\partial_{t}\right|_{t=0}} \widetilde{\nu}\right)\left(\xi_{1}, \ldots, \widetilde{\nabla}_{\left.\partial_{t}\right|_{t=0}} \widetilde{\xi}_{\rho}, \ldots, \xi_{r}\right)
\end{aligned}
$$




$$
\begin{aligned}
= & \widetilde{\nabla}_{\left.\partial_{t}\right|_{t=0}}\left(K\left(\frac{d \nu_{t}}{d t}\right)\left(\theta_{t}^{\perp-1}(1)\right)\right) \\
& -\sum_{\rho=1}^{r} K\left(\left.\frac{d \nu_{t}}{d t}\right|_{t=0}\right)\left(\xi_{1}, \ldots, \widetilde{\nabla}_{\left.\partial_{t}\right|_{t=0}} \widetilde{\xi}_{\rho}, \ldots, \xi_{r}\right) .
\end{aligned}
$$

Since $F_{t}(-\varepsilon<t<\varepsilon)$ are isometric immersions, we have $K\left(\frac{d \nu_{t}}{d t}\right)\left(\theta_{t}^{\perp-1}(1)\right)=0$. Hence we have

$$
\begin{aligned}
& K\left(\left.\frac{d}{d t}\right|_{t=0} K\left(\frac{d \nu_{t}}{d t}\right)\right)\left(\theta^{\perp-1}(1)\right) \\
& \quad=-\sum_{\rho=1}^{r} K\left(\left.\frac{d \nu_{t}}{d t}\right|_{t=0}\right)\left(\xi_{1}, \ldots,\left(\widetilde{\nabla}_{\left.\partial_{t}\right|_{t=0}} \widetilde{\xi}_{\rho}\right)_{T}, \ldots, \xi_{r}\right) .
\end{aligned}
$$

The right-hand side $(*)$ of this relation is rewritten as follows:

$$
\begin{aligned}
(*) & =-\sum_{\rho=1}^{r}\left(\widetilde{\nabla}_{\left.\partial_{t}\right|_{t=0}} \widetilde{\nu}\right)\left(\xi_{1}, \ldots,\left(\widetilde{\nabla}_{\left.\partial_{t}\right|_{t=0}} \widetilde{\xi}_{\rho}\right)_{T}, \ldots, \xi_{r}\right) \\
& =\sum_{\rho=1}^{r} \nu\left(\xi_{1}, \ldots, \widetilde{\nabla}_{\left.\partial_{t}\right|_{t=0}}\left(\left(\widetilde{\nabla}_{\partial_{t}} \widetilde{\xi}_{\rho}\right)_{T}\right), \ldots, \xi_{r}\right) \\
& =\sum_{\rho=1}^{r} \widetilde{g}\left(\widetilde{\nabla}_{\left.\partial_{t}\right|_{t=0}}\left(\left(\widetilde{\nabla}_{\partial_{t}} \widetilde{\xi}_{\rho}\right)_{T}\right), \xi_{\rho}\right) \\
& =-\sum_{\rho=1}^{r} \widetilde{g}\left(\left(\widetilde{\nabla}_{\left.\partial_{t}\right|_{t=0}} \widetilde{\xi}_{\rho}\right)_{T},\left(\widetilde{\nabla}_{\left.\partial_{t}\right|_{t=0}} \widetilde{\xi}_{\rho}\right)_{T}\right) \\
& =-\sum_{\rho=1}^{r}\left\|\left(\widetilde{\nabla}_{\left.\partial_{t}\right|_{t=0}} \widetilde{\xi}_{\rho}\right)_{T}\right\|_{\tilde{g}}^{2} .
\end{aligned}
$$

Let $\left(e_{1}^{t}, \ldots, e_{n}^{t}\right)$ be an orthonormal frame field of $f_{t *} T M$ and $\widetilde{e}_{i}(i=1, \ldots, n)$ be a section of $\delta^{*} T \widetilde{M}$ defined by $\widetilde{e}_{i}(x, t)=\left(e_{i}^{t}\right)_{x}((x, t) \in D \times(-\varepsilon, \varepsilon))$. On the other hand, we have

$$
\begin{aligned}
\left(\widetilde{\nabla}_{\left.\partial_{t}\right|_{t=0}} \widetilde{\xi}_{\rho}\right)_{T} & =\sum_{i=1}^{n} \widetilde{g}\left(\widetilde{\nabla}_{\left.\partial_{t}\right|_{t=0}} \widetilde{\xi}_{\rho}, e_{i}\right) e_{i} \\
& =-\sum_{i=1}^{n} \widetilde{g}\left(\xi_{\rho}, \widetilde{\nabla}_{\left.\partial_{t}\right|_{t=0}} \widetilde{e}_{i}\right) e_{i}
\end{aligned}
$$




$$
=-\sum_{i=1}^{n} \widetilde{g}\left(\xi_{\rho}, \widetilde{\nabla}_{e_{i}} \pi_{*} v+\left.\left[\delta_{*} \partial_{t}, \widetilde{e}_{i}\right]\right|_{t=0}\right) e_{i}
$$

It is easy to show that $\left.\left[\delta_{*} \partial_{t}, \widetilde{e}_{i}\right]\right|_{t=0} \in f_{*} T M$. Hence we have

$$
\left(\widetilde{\nabla}_{\left.\partial_{t}\right|_{t=0}} \widetilde{\xi}_{\rho}\right)_{T}=-\sum_{i=1}^{n} \widetilde{g}\left(\xi_{\rho}, h\left(e_{i},\left(\pi_{*} v\right)_{T}\right)+\nabla_{e_{i}}^{\perp}\left(\pi_{*} v\right)_{N}\right) e_{i} .
$$

From (3.9), (3.10) and this relation, the desired relation is deduced.

\section{Some classes consisting of non-degenerate equiaffine immersions}

In this section, we treat non-degenerate equiaffine immersions into a general equiaffine manifold. Note that the non-degeneracy in the sense of this paper is a property stricter than the non-degeneracy and the strictly non-degeneracy in the sense of $[\mathrm{KT}]$. Let $E_{N D}(M)$ be the space of all non-degenerate equiaffine immersions (in the sense of this paper) of a manifold $M$ into an equiaffine manifold $(\widetilde{M}, \widetilde{\nabla}, \widetilde{\theta})$. For each $F \in E_{N D}(M)$, we define a function $\mathrm{VR}_{F}$ over $M$ by $\omega_{h}=\mathrm{VR}_{F} \theta$, where $\theta$ is the induced volume element of $F$ and $\omega_{h}$ is the volume element defined from the affine fundamental form $h$ of $F$. Note that this function $\mathrm{VR}_{F}$ is positive-valued by the definition of $\omega_{h}$. We call this function $\mathrm{VR}_{F}$ the volume ratio function of $F$. For each positive function $\psi$ over $M$, we denote $\left\{F \in E_{N D}(M) \mid \mathrm{VR}_{F}=\psi\right\}$ by $V R^{-1}(\psi)$. Also, for each positive function $\psi$ over $M$, we denote $\left\{F \in E_{N D}(M) \mid \widehat{T}_{F}=d \log \psi\right\}$ by $\widehat{T}^{-1}(\psi)$, where $\widehat{T}_{F}$ is the Tchebychev form of $F$. Denote by $N D(M)$ the space of all non-degenerate immersions of $M$ into $(\widetilde{M}, \widetilde{\nabla}, \widetilde{\theta})$ in the sense of this paper. Define a map $\widehat{\pi}$ of $E_{N D}(M)$ onto $N D(M)$ by $\widehat{\pi}(F):=\pi \circ F$, where $\pi$ is the bundle projection of $\bigwedge^{r}(T \widetilde{M})$. For $f \in N D(M)$, denote by $\Gamma_{0}\left(f^{*} T^{*} \widetilde{M} \otimes T M\right)$ the set of all sections of $f^{*} T^{*} \widetilde{M} \otimes T M$ vanishing on $f_{*} T M$. For $\Psi \in \Gamma_{0}\left(f^{*} T^{*} \widetilde{M} \otimes T M\right)$, we denote $\left\{F \in \widehat{\pi}^{-1}(f) \mid \widetilde{T}_{F}=\Psi\right\}$ by $\widetilde{T}^{-1}(\Psi)$, where $\widetilde{T}_{F}$ is the extended Tchebychev tensor field of $F$. For these classes, we prove the following facts.

Theorem 4.1 Let $\operatorname{dim} M=n, \operatorname{dim} \widetilde{M}=n+r, f \in N D(M), \psi \in$ $C^{\infty}(M)_{+}$and $\Psi \in \Gamma_{0}\left(f^{*} T^{*} \widetilde{M} \otimes T M\right)$. Then the following statements (i) ( vi) hold:

(i) $\widehat{T}^{-1}(\psi)=\underset{c \in \mathbf{R}_{+}}{\cup} V R^{-1}\left(c \psi^{1 / 2 r}\right)$.

(ii) $\widehat{\pi}^{-1}(f) \cap V R^{-1}(\psi) \neq \emptyset$, 
(iii) Let $G_{f, x}\left(V R^{-1}(\psi)\right):=\left\{\left(N_{F}\right)_{x} \mid F \in \widehat{\pi}^{-1}(f) \cap V R^{-1}(\psi)\right\}$, where $N_{F}$ is the transversal bundle of $F$. Then $G_{f, x}\left(V R^{-1}(\psi)\right)$ are connected and of dimension at most $n(r-1)$ in the following sense:

For any $n(r-1)+1$-dimensional topologically embedded submanifold $L$ in the Grassmannian manifold $G_{r}\left(T_{f(x)} \widetilde{M}\right)$ of $r$-dimensional subspaces in $T_{f(x)} \widetilde{M}, L \cap G_{f, x}\left(V R^{-1}(\psi)\right)$ has no interior point as subsets of $L$.

(iv) For arbitrary two points $F$ and $G$ of $\widehat{\pi}^{-1}(f) \cap V R^{-1}(\psi)$, there exists a variation $F_{t}(0 \leq t \leq 1)$ in $\widehat{\pi}^{-1}(f) \cap V R^{-1}(\psi)$ with $F_{0}=F$ and $F_{1}=G$.

(v) $\widetilde{T}^{-1}(\Psi) \neq \emptyset$ and the set $G_{f, x}\left(\widetilde{T}^{-1}(\Psi)\right):=\left\{\left(N_{F}\right)_{x} \mid F \in \widetilde{T}^{-1}(\Psi)\right\}$ is an one-point set.

(vi) There exists $\psi_{0} \in C^{\infty}(M)_{+}$satisfying

$$
\sharp\left(\widetilde{T}^{-1}(\Psi) \cap V R^{-1}(\psi)\right)= \begin{cases}1 & \left(\psi \in \mathbf{R} \psi_{0}\right) \\ 0 & \left(\psi \notin \mathbf{R} \psi_{0}\right) .\end{cases}
$$

In particular, we have

$$
\begin{aligned}
& \sharp\left(\widetilde{T}^{-1}(0) \cap V R^{-1}(c)\right)=1 \quad(c \text { : a positive constant }) \\
& \sharp\left(\widetilde{T}^{-1}(0) \cap V R^{-1}(\psi)\right)=0 \quad(\psi \text { : a non-constant positive function }),
\end{aligned}
$$

where 0 is the zero element of $\Gamma_{0}\left(f^{*} T \widetilde{M} \otimes T M\right)$.

Proof. According to Proposition 2.2, we have $\widehat{T}=2 r d \log \frac{\omega_{h}}{\theta}$ for each $F \in$ $E_{N D}(M)$, where $\widehat{T}, \theta$ and $\omega_{h}$ are the quantities for $F$. Hence we have the relation of (i). Next we shall show the statements (ii) (iv). Let $F \in \widehat{\pi}^{-1}(f)$. Take another element $\bar{F}$ of $\widehat{\pi}^{-1}(f)$. Let $h, \nabla^{\perp}, \theta, T$ and $\hat{T}$ be the quantities for $F$ and $\bar{h}, \bar{\nabla}^{\perp}, \bar{\theta}, \bar{T}$ and $\overline{\hat{T}}$ be the quantities for $\bar{F}$. Let $U$ be a contractible open set of $M$. On $U$, we can express $F$ and $\bar{F}$ as $F=\xi_{1} \wedge \cdots \wedge \xi_{r}$ and $\bar{F}=\left(f_{*} X_{1}+\eta \xi_{1}\right) \wedge \cdots \wedge\left(f_{*} X_{r}+\eta \xi_{r}\right)$ in terms of a frame field $\left(\xi_{1}, \ldots, \xi_{r}\right)$ of $N_{F}$ on $U$ and tangnet vector fields $X_{1}, \ldots, X_{r}$ on $U$ and $\eta \in C^{\infty}(U)_{+}$. Set $\bar{\xi}_{\rho}:=f_{*} X_{\rho}+\eta \xi_{\rho}(\rho=1, \ldots, r)$. From (2.7) of [KT], we have $\bar{\tau}_{\rho}^{\rho}=\tau_{\rho}^{\rho}+$ $\eta^{-1}\left(h^{\rho}\left(X_{\rho}, \cdot\right)+r d \eta\right)$, where $\tau_{\rho}^{\gamma}$ (resp. $\left.\bar{\tau}_{\rho}^{\gamma}\right)$ is defined by $\nabla^{\perp} \xi_{\rho}=\tau_{\rho}^{\gamma} \otimes \xi_{\gamma}$ (resp. $\left.\bar{\nabla}^{\perp} \bar{\xi}_{\rho}=\bar{\tau}_{\rho}^{\gamma} \otimes \bar{\xi}_{\gamma}\right)$ and $h^{\rho}$ is defined by $h=h^{\rho} \otimes \xi_{\rho}$. From the equiaffinities of $F$ and $\bar{F}$, we have $\tau_{\rho}^{\rho}=\bar{\tau}_{\rho}^{\rho}=0$. Hence we have

$$
h^{\rho}\left(X_{\rho}, \cdot\right)=-r d \eta \text {. }
$$


Since $F$ is non-degenerate, we may assume that $h^{\rho}(\rho=1, \ldots, r)$ are non-degenerate by retaking $\left(\xi_{1}, \ldots, \xi_{r}\right)$ if necessary. Hence, for each $\eta$, there exist tangent vector fields $X_{1}, \ldots, X_{n}$ on $U$ satisfying (4.1). On the other hand, we have $\omega_{\bar{h}} / \bar{\theta}=\eta^{-(n+2 r) / 2} \times \omega_{h} / \theta$ (see the proof of Lemma 5.2 in [Wi]). Hence $\bar{F} \in V R^{-1}(\psi)$ if and only if $\eta=\psi^{-2 /(n+2 r)} \times\left(\omega_{h} / \theta\right)^{2 /(n+2 r)}$ and (4.1) hold. From these facts, it follows that the statements (ii), (iii) and (iv) hold for $\left.f\right|_{U}$. Hence these statements hold for $f$, where we note that the simply connectedness of $M$ does not need to be assumed because it follows from the above investigation that $\left\{F(x) \mid F \in \widehat{\pi}^{-1}(f) \cap V R^{-1}(\psi)\right\}(x \in M)$ is connected. Next we shall show the statement $(\mathrm{v})$. Let $F \in \widehat{\pi}^{-1}(f)$. Take another $\bar{F} \in \widehat{\pi}^{-1}(f)$. Let $U, \xi_{\rho}, \bar{\xi}_{\rho}, X_{\rho}(\rho=1, \ldots, r)$ and $\eta$ be as in the proof of (ii), (iii) and (iv). From the equiaffinities of $F$ and $\bar{F}$, we have the relation (4.1). From (5.3) of [Wi], $\bar{F} \in \widetilde{T}_{\Psi}$ holds if and only if

$$
\eta\left(T_{\rho}^{i}+(n+r) X_{\rho}^{i}+h_{j k}^{\gamma} \mathfrak{h}_{\rho}^{i j} X_{\gamma}^{k}\right)=\bar{\Psi}_{\rho}^{i}=\eta \Psi_{\rho}^{i},
$$

that is,

$$
(n+r) X_{\rho}^{i}+h_{j k}^{\gamma} \mathfrak{h}_{\rho}^{i j} X_{\gamma}^{k}=\Psi_{\rho}^{i}-T_{\rho}^{i},
$$

where $X_{\rho}^{i}, h_{i j}^{\rho}, \mathfrak{h}_{\rho}^{i j}, \Psi_{\rho}^{i}$ and $T_{\rho}^{i}$ are the components of $X_{\rho}, h, \mathfrak{h}, \Psi$ and $T$ with respect to a local coordinate $\left(x^{1}, \ldots, x^{n}\right)$ on $U$ and $\left(\xi_{1}, \ldots, \xi_{r}\right)$. Since (4.2) is a simultaneously linear equation with respect to $n r$ pieces of unknown variables $X_{\rho}^{i}(\rho=1, \ldots, r, i=1, \ldots, n)$ consisting $n r$ pieces of equations, it has at least one solution. For each solution $X_{\rho}^{i}$ of (4.2), there exists a positive function $\eta$ on $U$ satisfying (4.1). These facts imply $\widetilde{T}^{-1}\left(\left.\Psi\right|_{U}\right) \neq \emptyset$. Hence $\widetilde{T}^{-1}(\Psi) \neq \emptyset$ follows. Assume that $F, \bar{F} \in \widetilde{T}^{-1}(\Psi)$. From (5.3) of [Wi], we have

$$
\bar{T}_{\rho}^{i}-\eta T_{\rho}^{i}=\eta\left((n+r) X_{\rho}^{i}+h_{j k}^{\gamma} \mathfrak{h}_{\rho}^{i j} X_{\gamma}^{k}\right) .
$$

On the other hand, we have

$$
\bar{T}_{\rho}^{i}-\eta T_{\rho}^{i}=\overline{\widetilde{T}}\left(\bar{\xi}_{\rho}\right)^{i}-\widetilde{T}\left(\eta \xi_{\rho}\right)^{i}=\Psi\left(\bar{\xi}_{\rho}-\eta \xi_{\rho}\right)^{i}=\Psi\left(f_{*} X_{\rho}\right)^{i}=0 .
$$

Hence we have

$$
(n+r) X_{\rho}^{i}+h_{j k}^{\gamma} \mathfrak{h}_{\rho}^{i j} X_{\gamma}^{k}=0,
$$


that is, $X_{\rho}^{i}=-(1 /(n+r)) h_{j k}^{\gamma} \mathfrak{h}_{\rho}^{i j} X_{\gamma}^{k}$. Furthermore, we have

$$
\begin{aligned}
(n+r) X_{\rho}^{i}+h_{j k}^{\gamma} \mathfrak{h}_{\rho}^{i j} X_{\gamma}^{k} & =(n+r) X_{\rho}^{i}+h_{j k}^{\gamma} \mathfrak{h}_{\rho}^{i j}\left(-\frac{1}{n+r}\right) h_{l m}^{\mu} \mathfrak{h}_{\gamma}^{k l} X_{\mu}^{m} \\
& =(n+r) X_{\rho}^{i}-\frac{r}{n+r} \mathfrak{h}_{\rho}^{i l} h_{l m}^{\mu} X_{\mu}^{m}=0,
\end{aligned}
$$

that is, $(n+r) X_{\rho}^{i}-(r /(n+r)) h_{j k}^{\gamma} \mathfrak{h}_{\rho}^{i j} X_{\gamma}^{k}=0$. This together with (4.3) deduces $X_{\rho}=0$, which implies $N=\bar{N}$. Thus $G_{f, x}\left(\widetilde{T}^{-1}(\Psi)\right)$ is a one point set.

Next we shall show the statement (vi). Take $\psi \in C^{\infty}(M)$. Assume that $F, \bar{F} \in \widetilde{T}^{-1}(\Psi) \cap V R^{-1}(\psi)$. By (v), we have $N=\bar{N}$. Since $F, \bar{F} \in V R^{-1}(\psi)$, we have $\omega_{h} / \theta=\omega_{\bar{h}} / \bar{\theta}(=\psi)$. These facts imply $\theta=\bar{\theta}$, that is, $F=\bar{F}$. Hence we have $\sharp\left(\widetilde{T}^{-1}(\Psi) \cap V R^{-1}(\psi)\right) \leq 1$. It is clear that $\sharp\left(\widetilde{T}^{-1}(\Psi) \cap V R^{-1}\left(\psi_{0}\right)\right)$ $=1$ for some $\psi_{0} \in C^{\infty}(M)_{+}$. Assume that $\sharp\left(\widetilde{T}^{-1}(\Psi) \cap V R^{-1}\left(\psi_{1}\right)\right)=1$ for another $\psi_{1} \in C^{\infty}(M)_{+}$. Let $F_{i} \in \widetilde{T}^{-1}(\Psi) \cap V R^{-1}\left(\psi_{i}\right)(i=0,1)$. Also, let $N_{i}$ (resp. $\theta_{i}^{\perp}$ ) be the transversal bundle (resp. the transversal volume element) of $F_{i}$. By (v), we have $N_{0}=N_{1}$. Hence it follows from the equiaffinities of $F_{i}(i=0,1)$ that $\theta_{0}^{\perp}=c \theta_{1}^{\perp}$ for some positive constant $c$. This implies that $\psi_{0}=c^{(n+2 r) /(2 r)} \psi_{1}$. Hence we have

$$
\sharp\left(\widetilde{T}^{-1}(\Psi) \cap V R^{-1}(\psi)\right)= \begin{cases}1 & \left(\psi \in \mathbf{R} \psi_{0}\right) \\ 0 & \left(\psi \notin \mathbf{R} \psi_{0}\right) .\end{cases}
$$

It is clear that

$$
\widetilde{T}^{-1}(0) \cap V R^{-1}(\psi)=\left\{F \in \widehat{\pi}^{-1}(f) \cap V R^{-1}(\psi) \mid T_{F}=0\right\},
$$

where $T_{F}$ is the Tchebychev tensor field of $F$. Hence, if $\widetilde{T}^{-1}(0) \cap V R^{-1}(\psi) \neq$ $\emptyset$, then we have $d \log \psi=0$ by Proposition 2.2. That is, $\psi$ is constant. Hence we have $\sharp\left(\widetilde{T}^{-1}(0) \cap V R^{-1}(c)\right)=1$ and $\sharp\left(\widetilde{T}^{-1}(0) \cap V R^{-1}(\psi)\right)=0$ for each non-constant positive function $\psi$.

According to (vi) of this theorem, there exists the only element of $V R^{-1}(c) \cap \widehat{\pi}^{-1}(f)$ having vanishing Tchebychev tensor field for each $f \in$ $N D(M)$ and each positive constant $c$. We denote this element by $f_{c B}$ and call it a $c$-Blaschke immersion (associated with $f$ ). In particular, when $c=1$, we denote it by $f_{B}$ and call it the Blaschke immersion (associated with $f$ ). For example, for any homogeneous isometric embedding with parallel second fundamental form (i.e., the inclusion map of homogeneous 
pseudo-Riemannian submanifold with parallel second fundamental form) $f: M \hookrightarrow \widetilde{M}$, an immersion $F: M \hookrightarrow \bigwedge^{r} T \widetilde{M}$ defined by $F(x)=\xi_{1}^{x} \wedge \cdots \wedge \xi_{r}^{x}$ $\left(\left(\xi_{1}^{x}, \ldots, \xi_{r}^{x}\right)\right.$ : an orthonormal normal base of $f$ at $\left.x\right)$ for $x \in M$ is $c$-Blaschke one for some $c>0$.

\section{The variational formulas for some volume functions over the space of all non-degenerate immersions}

For an immersion $\phi$ of $M \backslash D$ (D: a relatively compact domain with smooth boundary of $M)$ into $\widetilde{M}$, we define $\left.E_{N D}(M)_{\phi}\right|_{D},\left.V R^{-1}(\psi)_{\phi}\right|_{D}$, $\left.\widehat{T}^{-1}(\psi)_{\phi}\right|_{D}$ and $\left.\widetilde{T}^{-1}(\Psi)_{\phi}\right|_{D}$ in similar to $\left.E(M)_{\phi}\right|_{D}$.

Theorem 5.1 Let $\left.F \in V R^{-1}(\psi)_{\phi}\right|_{D}, F_{t}(-\varepsilon<t<\varepsilon)$ be a variation of $F$ in $\left.V R^{-1}(\psi)_{\phi}\right|_{D}$ and $\nu_{t}$ be the transversal volume element map of $F_{t}$, where $\psi$ is a positive function on $M$ and $\phi$ is a non-degenerate immersion of $M \backslash \stackrel{D}{D}$ $(D:$ a compact domain of $M)$ into $\bigwedge^{r}(T \widetilde{M})$. Let $v:=\left.\frac{d F_{t}}{d t}\right|_{t=0}$. Then we have

$$
\begin{aligned}
\left.\frac{d}{d t}\right|_{t=0} \operatorname{Vol}\left(F_{t}\right) & \\
=-\frac{1}{n+2 r} & \int_{D}\left(n(n+r) H\left(\left(\pi_{*} v\right)_{N}\right)-\mu_{\widetilde{R}, \mathfrak{h}}\left(\pi_{*} v\right)\right. \\
& \left.+2 r(d \log \psi)\left(\left(\pi_{*} v\right)_{T}\right)+\operatorname{tr}\left(T \circ \nabla^{\perp}\left(\pi_{*} v\right)_{N}\right)\right) \theta
\end{aligned}
$$

Here $\theta$ is the induced volume element of $F, H$ is the affine mean curvature function of $F, T$ is the Tchebychev tensor field of $F, \nabla^{\perp}$ is the transversal connection of $F, h$ is the affine fundamental form of $F$ and $\pi$ is the bundle projection of $\bigwedge^{r} T \widetilde{M},(\cdot)_{T}$ (resp. $\left.(\cdot)_{N}\right)$ is the tangential (resp. transversal) component of $(\cdot)$ and $\mu_{\widetilde{R}, \mathfrak{h}}$ is a section of $f^{*} T^{*} \widetilde{M}$ defined by $\mu_{\widetilde{R}, \mathfrak{h}}(\cdot):=$ $\operatorname{tr} \widetilde{R}\left(\cdot, f_{*} \partial_{i}, f_{*} \partial_{j}\right)_{N} \mathfrak{h}^{i j}$ in terms of a local coordinate $\left(x^{1}, \ldots, x^{n}\right)$ of $M$, where $\widetilde{R}$ is the curvature tensor of $\widetilde{\nabla}, f:=\pi \circ F, \partial_{i}:=\partial / \partial x^{i}$ and $\mathfrak{h}(\cdot)=$ $\mathfrak{h}^{i j}(\cdot) \partial_{i} \otimes \partial_{j}(\mathfrak{h}$ : the pseudo-inverse of $h)$.

Proof. Let $F, F_{t}(-\varepsilon<t<\varepsilon)$ and $v$ be as in the statement. Let $N_{t}$, $h_{t}, \widetilde{h}_{t}, \mathfrak{h}_{t}, \theta_{t}$ and $\theta_{t}^{\perp}$ be the quantities for $F_{t}$. In particular, set $N:=N_{0}$, $h=h_{0}, \widetilde{h}=\widetilde{h}_{0}, \mathfrak{h}:=\mathfrak{h}_{0}, \theta:=\theta_{0}$ and $\theta^{\perp}:=\theta_{0}^{\perp}$. Let $\left(U,\left(x^{1}, \ldots, x^{n}\right)\right)$ be a local coordinate of $M$ with $U \subset D$ and $\left(\xi_{1}^{t}, \ldots, \xi_{r}^{t}\right)$ be a local frame field of $N_{t}$ over $U$ such that $\theta_{t}^{\perp}\left(\xi_{1}^{t}, \ldots, \xi_{r}^{t}\right)=1$ and that $\xi_{i}^{t}(i=1, \ldots, r)$ are smooth with respect to $t$. Let $h_{t}\left(\partial_{i}, \partial_{j}\right)=h_{i j}^{\rho}(t) \xi_{\rho}^{t}$ and $\mathfrak{h}_{t}\left(\xi_{\rho}^{t}\right)=\mathfrak{h}_{\rho}^{i j}(t) \partial_{i} \otimes \partial_{j}$ 
$(i, j=1, \ldots, n)$. Also, let $\partial_{t}=\partial / \partial t$. According to the proof of (iii) of Lemma 4.13 of [Wi], we have

$$
\left.\partial_{t}\right|_{t=0} \omega_{h_{t}}\left(\partial_{1}, \ldots, \partial_{n}\right)^{2 r}=\left(\left.\partial_{t}\right|_{t=0} h_{i j}^{\rho}\right) \mathfrak{h}_{\rho}^{i j}(0) \omega_{h}\left(\partial_{1}, \ldots, \partial_{n}\right)^{2 r}
$$

and hence

$$
\left.\partial_{t}\right|_{t=0} \omega_{h_{t}}\left(\partial_{1}, \ldots, \partial_{n}\right)=\frac{1}{2 r}\left(\left.\partial_{t}\right|_{t=0} h_{i j}^{\rho}\right) \mathfrak{h}_{\rho}^{i j} \omega_{h}\left(\partial_{1}, \ldots, \partial_{n}\right) .
$$

Define a section $\omega_{t}^{\rho}$ of $f_{t}^{*} T^{*} \widetilde{M}$ by $\omega_{t}^{\rho}\left(\xi_{\gamma}^{t}\right)=\delta_{\gamma}^{\rho}$ and $\operatorname{Ker} \omega_{t}^{\rho}=f_{t *} T M$. According to (6.6) of [Wi], we have

$$
\begin{aligned}
\left.\partial_{t}\right|_{t=0} h_{i j}^{\rho}= & -h_{i j}^{\gamma} \omega^{\rho}\left(\widetilde{\nabla}_{\left.\partial_{t}\right|_{t=0}} \xi_{\gamma}^{t}\right)-\Gamma_{i j}^{k} \omega^{\rho}\left(\widetilde{\nabla}_{\left.\partial_{t}\right|_{t=0}} \delta_{*} \partial_{k}\right) \\
& +\omega^{\rho}\left(\widetilde{\nabla}_{\left.\partial_{t}\right|_{t=0}} \widetilde{\nabla}_{\partial_{i}} \delta_{*} \partial_{j}\right),
\end{aligned}
$$

where $\omega^{\rho}:=\omega_{0}^{\rho}$ and $\Gamma_{i j}^{k}$ is defined by $\nabla_{\partial_{i}} \partial_{j}=\Gamma_{i j}^{k} \partial_{k}$. Let $B_{\pi_{*} v}:=$ $h\left(\left(\pi_{*} v\right)_{T}, \cdot\right)+\nabla^{\perp}\left(\pi_{*} v\right)_{N}$ and define $\bar{B}_{\pi_{*} v}$ by $\bar{B}_{\pi_{*} v}^{i}=\mathfrak{h}_{\rho}^{i j}(0)\left(B_{\pi_{*} v}\right)_{j}^{\rho}$. From (5.2) and (5.3), we have

$$
\begin{aligned}
\left.2 r \partial_{t}\right|_{t=0} \log \omega_{h_{t}}\left(\partial_{1}, \ldots, \partial_{n}\right) \\
=\left(-h_{i j}^{\rho} \omega^{\gamma}\left(\widetilde{\nabla}_{\left.\partial_{t}\right|_{t=0}} \xi_{\rho}^{t}\right)-\Gamma_{i j}^{k} \omega^{\gamma}\left(\widetilde{\nabla}_{\left.\partial_{t}\right|_{t=0}} \delta_{*} \partial_{k}\right)+\omega^{\gamma}\left(\widetilde{\nabla}_{\left.\partial_{t}\right|_{t=0}} \widetilde{\nabla}_{\partial_{i}} \delta_{*} \partial_{j}\right)\right) \mathfrak{h}_{\gamma}^{i j} \\
=n K\left(v_{\nu}\right)\left(\theta^{\perp-1}(1)\right)-\mathfrak{h}_{\gamma}^{i j} \Gamma_{i j}^{k}\left(B_{\pi_{*} v}\right)_{k}^{\gamma} \\
\quad+\mathfrak{h}_{\gamma}^{i j} \omega^{\gamma}\left(\widetilde{R}\left(\pi_{*} v, f_{*} \partial_{i}\right) f_{*} \partial_{j}+\widetilde{\nabla}_{\partial_{i}} \widetilde{\nabla}_{\partial_{t}} \delta_{*} \partial_{j}\right) .
\end{aligned}
$$

By somewhat long calculations, it is shown that the sum of the second and third terms is equal to

$$
\begin{aligned}
& r \operatorname{div}_{\nabla}\left(\pi_{*} v\right)_{T}-n r H\left(\left(\pi_{*} v\right)_{N}\right)+\operatorname{div}_{\nabla} \bar{B}_{\pi_{*} v} \\
& -\operatorname{tr}\left(T \circ B_{\pi_{*} v}\right)+\mu_{\widetilde{R}, \mathfrak{h}}\left(\pi_{*} v\right) .
\end{aligned}
$$

Hence we have

$$
\begin{aligned}
\left.\partial_{t}\right|_{t=} & \omega_{h_{t}}\left(\partial_{1}, \ldots, \partial_{n}\right) \\
= & \frac{1}{2 r}\left(n K\left(v_{\nu}\right)\left(\theta^{\perp-1}(1)\right)+r \operatorname{div}_{\nabla}\left(\pi_{*} v\right)_{T}-n r H\left(\left(\pi_{*} v\right)_{N}\right)\right. \\
& \left.+\operatorname{div}_{\nabla} \bar{B}_{\pi_{*} v}-\operatorname{tr}\left(T \circ B_{\pi_{*} v}\right)+\mu_{\widetilde{R}, \mathfrak{h}}\left(\pi_{*} v\right)\right) \omega_{h}\left(\partial_{1}, \ldots, \partial_{n}\right) .
\end{aligned}
$$

On the other hand, we have

$$
\left.\partial_{t}\right|_{t=0} \theta_{t}\left(\partial_{1}, \ldots, \partial_{n}\right)=-\left(K\left(v_{\nu}\right)\left(\theta^{\perp-1}(1)\right)+n H\left(\left(\pi_{*} v\right)_{N}\right)\right.
$$




$$
\left.-\operatorname{div}_{\nabla}\left(\left(\pi_{*} v\right)_{T}\right)\right) \theta\left(\partial_{1}, \ldots, \partial_{n}\right)
$$

(see the proof of (i) of Theorem 3.1). Also, we have

$$
\left.\partial_{t}\right|_{t=0} \omega_{h_{t}}\left(\partial_{1}, \ldots, \partial_{n}\right)=\left.\frac{\omega_{h}}{\theta} \partial_{t}\right|_{t=0} \theta_{t}\left(\partial_{1}, \ldots, \partial_{n}\right)
$$

because $\left.\partial_{t}\right|_{t=0} \omega_{h_{t}} / \theta_{t}=0$ by the assumption. From (5.4) (5.6), we have

$$
\begin{aligned}
K\left(v_{\nu}\right)\left(\theta^{\perp-1}(1)\right)= & \frac{1}{n+2 r}\left\{r \operatorname{div}_{\nabla}\left(\pi_{*} v\right)_{T}-n r H\left(\left(\pi_{*} v\right)_{N}\right)\right. \\
& \left.-\operatorname{div}_{\nabla} \bar{B}_{\pi_{*} v}+\operatorname{tr}\left(T \circ B_{\pi_{*} v}\right)-\mu_{\widetilde{R}, \mathfrak{h}}\left(\pi_{*} v\right)\right\} .
\end{aligned}
$$

Also, we have $\operatorname{tr}\left(T \circ B_{\pi_{*} v}\right)=\widehat{T}\left(\left(\pi_{*} v\right)_{T}\right)+\operatorname{tr}\left(T \circ \nabla^{\perp}\left(\pi_{*} v\right)_{N}\right)$. By substituting (5.7) and this relation into the first variational formula in (i) of Theorem 3.1, we obtain the desired relation, where we also use Proposition 2.2 .

For an immersion $\phi$ of $M \backslash D$ (D: a relatively compact domain with smooth boundary of $M$ ) into $\widetilde{M}$, we set $N D(M)_{\phi}:=\{f \in N D(M) \mid f=\phi$ on $M \backslash D\}$ and $\left.N D(M)_{\phi}\right|_{D}:=\left\{\left.f\right|_{D} \mid f \in N D(M)_{\phi}\right\}$. We define the function $\operatorname{Vol}_{C B, D}$ on $\left.N D(M)_{\phi}\right|_{D}$ by

$$
\operatorname{Vol}_{c B, D}(f):=\operatorname{Vol}\left(f_{c B}\right) \quad\left(\left.f \in N D(M)_{\phi}\right|_{D}\right) .
$$

In particular, we have the following first variational formula for $c$-Blaschke immersions.

Corollary 5.2 Let $\left.f \in N D(M)_{\phi}\right|_{D}, f_{t}(-\varepsilon<t<\varepsilon)$ be a variation of $f$ in $\left.N D(M)_{\phi}\right|_{D}$ and $v:=\left.\frac{d f_{t}}{d t}\right|_{t=0}$. Then we have

$$
\left.\frac{d}{d t}\right|_{t=0} \operatorname{Vol}_{c B, D}\left(f_{t}\right)=-\frac{1}{n+2 r} \int_{D}\left(n(n+r) H\left(v_{N}\right)-\mu_{\widetilde{R}, \mathfrak{h}}(v)\right) \theta,
$$

where $H$ and $\mathfrak{h}$ are the quantities for $f_{c B}$.

Remark 5.1 (i) The above first variational formula for the case of $(\widetilde{M}, \widetilde{\nabla}, \widetilde{\theta})=\left(\mathbf{R}^{n+r}, \widetilde{\nabla}, \widetilde{\omega}\right)$ coincides with (the equiaffine case of) the formula (6.10) of [Wi].

(ii) in case of $\widetilde{R}\left(f_{*} T M, f_{*} T M\right) f_{*} T M \subset f_{*} T M$, we have $\mu_{\widetilde{R}, \mathfrak{h}}(v)=$ $\mu_{\widetilde{R}, \mathfrak{h}}\left(v_{N}\right)$. Hence, according to the above first variational formula, in the case where $f$ is a critical point of the volume function $\operatorname{Vol}_{c B, D}$, the index of the critical point $f$ is analyzed by investigating $\left.\frac{d^{2}}{d t^{2}}\right|_{t=0} \operatorname{Vol}_{c B, D}\left(f_{t}\right)$ for transversal variations $f_{t}$ of $f$ in $\left.N D(M)_{\phi}\right|_{D}$. 
From the first variational formula of Theorem 5.1 and (iv) of Theorem 4.1, the following fact follows.

Theorem 5.3 Let $F,\left.G \in V R^{-1}(\psi)_{\phi}\right|_{D}$. If $\pi \circ F=\pi \circ G$, then $\operatorname{Vol}(F)=$ $\operatorname{Vol}(G)$ holds.

Proof. Assume that $\pi \circ F=\pi \circ G$. Let $f:=\pi \circ F$. According to the statement (iv) of Theorem 4.1, there exists a variation $F_{t}(0 \leq t \leq 1)$ in $\left.\widehat{\pi}^{-1}(f) \cap V R^{-1}(\psi)_{\phi}\right|_{D}$ with $F_{0}=F$ and $F_{1}=G$. From Theorem 5.1, it follows that $(d / d t) \operatorname{Vol}\left(F_{t}\right)=0$ because $\pi \circ F_{t}=f(0 \leq t \leq 1)$. Hence we have $\operatorname{Vol}(F)=\operatorname{Vol}(G)$.

According to Theorem 5.3, for each positive function $\psi$ over $M$ and each relatively compact domain $D$ with smooth boundary of $M$, a function over $\left.N D(M)_{\phi}\right|_{D}$ is well-defined by assigning $\operatorname{Vol}\left(\left.F\right|_{D}\right)$ to each $\left.f \in N D(M)_{\phi}\right|_{D}$, where $F$ is an arbitrary element of $V R^{-1}(\psi)$ with $\left.(\pi \circ F)\right|_{D}=f$. We denote this function by $\operatorname{Vol}_{\psi, D}$. Here we note that $\operatorname{Vol}_{c, D}$ coincides with the above $\operatorname{Vol}_{c B, D}$. The following fact for $\mathrm{Vol}_{\psi, D}$ directly follows from Theorem 5.1.

Theorem 5.4 Let $\left.f \in N D(M)_{\phi}\right|_{D}$. Then $f$ is a critical point of $\operatorname{Vol}_{\psi, D}$ if and only if the following conditions $(a) \sim(c)$ hold on $D$ :

(a) $n(n+r) H-\left.\mu_{\widetilde{R}, \mathfrak{h}}\right|_{N}=0$,

(b) $\mu_{\widetilde{R}, \mathfrak{h}} \circ f_{*}=2 r d \log \psi$,

(c) $\operatorname{tr}(\bar{\nabla} T)=0$,

where $N, \mu_{\widetilde{R}, \mathfrak{h}}, H$ and $T$ are the quantities for an arbitrary element $F$ of $\left.V R^{-1}(\psi)_{\phi}\right|_{D}$ with $\pi \circ F=f$ and $\bar{\nabla}:=\nabla \otimes \nabla^{\perp *}\left(\nabla, \nabla^{\perp}\right.$ : the quantities for $F)$.

Proof. Let $f$ and $F$ be as in the statement. According to the first variational formula of Theorem 5.1, $\left.f\right|_{D}$ is a critical point of $\mathrm{Vol}_{\psi, D}$ if and only if for any $w \in \Gamma\left(f^{*} T \widetilde{M}\right)$ with supp $w \subset D$,

$$
\int_{D}\left(n(n+r) H\left(w_{N}\right)-\mu_{\widetilde{R}, \mathfrak{h}}(w)+2 r(d \log \psi)\left(w_{T}\right)+\operatorname{tr}\left(T \circ \nabla^{\perp} w_{N}\right)\right) \theta
$$

vanishes. Furthermore this holds if and only if the conditions $(a)$ and $(b)$ in the statement of Theorem 5.4 and the following condition $\left(c^{\prime}\right)$ hold:

$$
\left(c^{\prime}\right) \quad \int_{D} \operatorname{tr}\left(T \circ \nabla^{\perp} \xi\right) \theta=0 \text { for any } \xi \in \Gamma(N) \text { with } \operatorname{supp} \xi \subset D .
$$

We show that the condition $\left(c^{\prime}\right)$ is equivalent to the condition $(c)$ in the 
statement. For $\eta \in \Gamma(N)$, we have

$$
\operatorname{tr}\left(T \circ \nabla^{\perp} \eta\right)=\operatorname{div}_{\nabla} T(\eta)-\operatorname{tr}(\bar{\nabla} T)(\eta) .
$$

Hence, by using the Green's theorem, it is shown that the condition $(c)$ implies the condition $\left(c^{\prime}\right)$. We show the converse. Suppose $\operatorname{tr}(\bar{\nabla} T)_{x_{0}} \neq 0$ for some $x_{0} \in D$. Take a local section $\xi$ of $N$ over a sufficiently small neighborhood $U$ of $x_{0}$ in $D$ such that $\operatorname{tr}(\bar{\nabla} T)(\xi)$ has no zero point on $U$. Let $\rho$ be a positive function on $M$ with $\rho\left(x_{0}\right)=1$ and supp $\rho \subset U$. Clearly we have $\int_{D} \rho \operatorname{tr}(\bar{\nabla} T)(\xi) \theta \neq 0$. On the other hand, from the condition $\left(c^{\prime}\right)$, we have $\int_{D} \operatorname{tr}\left(T \circ \nabla^{\perp}(\rho \xi)\right) \theta=0$. This together with (5.8) and the Green's theorem deduces $\int_{D} \rho \operatorname{tr}(\bar{\nabla} T)(\xi) \theta=0$. Thus a contradiction arises. Hence it follows that $\operatorname{tr}(\nabla T)=0$ on $D$. Thus the condition $\left(c^{\prime}\right)$ implies the condition $(c)$. This completes the proof.

From this theorem, we have the following corollaries.

Corollary 5.5 Assume that $\widetilde{M}$ is flat. Let $\left.f \in N D(M)_{\phi}\right|_{D}$. Then $f$ is a critical point of $\operatorname{Vol}_{c, D}$ (c: a positive constant) if and only if $H=0$ holds on $D$, where $H$ is the affine mean curvature function of $f_{c B}$.

Corollary 5.6 Assume that $\widetilde{M}$ is flat. If $\psi$ is not constant on $D$, then $\mathrm{Vol}_{\psi, D}$ has no critical point.

Remark 5.2 According to Corollary 5.6, in the case where $\widetilde{M}$ is flat, we should treat $\operatorname{Vol}_{c, D}$ 's ( $c$ : a positive constant) as a volume function over $\left.N D(M)_{\phi}\right|_{D}$.

Also, we prove the following second variational formula.

Theorem 5.7 Assume that $\widetilde{M}$ is flat. Let $f$ be a critical point of $\operatorname{Vol}_{c, D}$. Then, for a transversal variation $f_{t}(-\varepsilon<t<\varepsilon)$ of $f$ in $\left.N D(M)_{\phi}\right|_{D}$, we have

$$
\begin{aligned}
\left.\frac{d^{2}}{d t^{2}}\right|_{t=0} \operatorname{Vol}_{c, D}\left(f_{t}\right)= & \frac{-1}{n+2 r} \int_{D}\left\{(n+r) \operatorname{tr}\left(A_{v}^{2}\right)-\frac{1}{n+2 r}\left(\triangle_{\mathfrak{h}} v\right)^{2}\right. \\
& -\operatorname{tr}\left(A_{v} \circ \nabla \bar{B}_{v}\right)+(n+r) \operatorname{tr}\left(Q_{v} \circ \nabla^{\perp} v\right) \\
& \left.+\operatorname{tr}\left(Q_{v} \circ h\left(\bar{B}_{v}, \cdot\right)\right)\right\} \theta,
\end{aligned}
$$

where $v:=\left.\frac{d f_{t}}{d t}\right|_{t=0}, A, h, \mathfrak{h}, \theta, \nabla$ and $\nabla^{\perp}$ are the quantities for $f_{c B}$, $\triangle_{\mathfrak{h}} v$ is defined by $\triangle_{\mathfrak{h}} v:=\mathfrak{h}_{\rho}^{i j}\left(\nabla_{\partial_{i}}^{\perp}\left(\nabla_{\partial_{j}}^{\perp} v\right)-\nabla_{\nabla_{\partial_{i}} \partial_{j}}^{\perp} v\right)^{\rho}$ in terms of the local 
expression, $\bar{B}_{v}$ is the tangent vector field of $D$ defined by $\bar{B}_{v}^{i}:=\mathfrak{h}_{\rho}^{i j} \nabla_{j}^{\perp} v^{\rho}$ in terms of the local expression and $Q_{v}$ is a section of the bundle $N^{*} \otimes T M$ $\left(N\right.$ : the transversal bundle of $\left.f_{c B}\right)$ defined as below.

Let $f, f_{t}, v$ and $N$ be as in the statement of Theorem 5.7. Define a map $\delta$ of $D \times(-\varepsilon, \varepsilon)$ into $\widetilde{M}$ by $\delta(x, t)=f_{t}(x)$. Take $\xi \in N_{x}$. Let $\widetilde{\xi}$ be a vector field along $t \rightarrow \delta(x, t)$ with $\widetilde{\xi}_{0}=\xi$ and $\widetilde{\xi}_{t} \in\left(N_{t}\right)_{x}$, where $N_{t}$ is the transversal bundle of $\left(f_{t}\right)_{c B}$. Then we define $Q_{v}(\xi)$ by $Q_{v}(\xi):=\left(\left.\widetilde{\nabla}_{\partial_{t}}\right|_{t=0} \widetilde{\xi}\right)_{T}$, which is independent of the choices of the variation $f_{t}$ with $\left.\frac{d f_{t}}{d t}\right|_{t=0}=v$ (because $f$ is a critical point of $\operatorname{Vol}_{c, D}$ ) and the extension $\widetilde{\xi}$ of $\xi$, that is, determined by only $v$ and $\xi$. Thus a section $Q_{v}$ of $N^{*} \otimes T M$ is well-defined by assigning $Q_{v}(\xi)$ to each $\xi \in N$.

Proof of Theorem 5.7. Since $f$ is a critical point of $\operatorname{Vol}_{c, D}, f_{t}$ is a transversal variation of $f$ and $\widetilde{M}$ is flat, it follows from (ii) of Theorem 3.1 and Theorem 5.4 that $H$ vanishes and

$$
\begin{aligned}
\left.\frac{d^{2}}{d t^{2}}\right|_{t=0} \operatorname{Vol}_{c, D}\left(f_{t}\right)=\int_{D} & \left\{2 K\left(\widetilde{v}_{\nu}\right)\left(\theta^{\perp-1}(1)\right)^{2}-\operatorname{tr}\left(A_{v}^{2}\right)\right. \\
& \left.-K\left(\left.\frac{d}{d t}\right|_{t=0} K\left(\frac{d \nu_{t}}{d t}\right)\right)\left(\theta^{\perp-1}(1)\right)\right\} \theta,
\end{aligned}
$$

where $H, A, \theta$ and $\theta^{\perp}$ are the quantities for $f_{c B}, v:=\left.\frac{d f_{t}}{d t}\right|_{t=0}$ and $\widetilde{v}_{\nu}:=$ $\left.\frac{d \nu_{t}}{d t}\right|_{t=0}\left(\nu_{t}\right.$ : the transversal volume element map of $\left.\left(f_{t}\right)_{c B}\right)$. From (5.7), we have

$$
K\left(\widetilde{v}_{\nu}\right)\left(\theta^{\perp-1}(1)\right)=-\frac{1}{n+2 r} \operatorname{div}_{\nabla} \bar{B}_{v}
$$

and

$$
\begin{aligned}
& K\left(\frac{d \nu_{t}}{d t}\right)\left(\theta_{t}^{\perp-1}(1)\right) \\
& \quad=\frac{1}{n+2 r}\left(r \operatorname{div}_{\nabla^{t}}\left(\delta_{*} \partial_{t}\right)_{T}-n r H_{t}\left(\left(\delta_{*} \partial_{t}\right)_{N}\right)-\operatorname{div}_{\nabla^{t}} \bar{B}_{\delta_{*} \partial_{t}}^{t}\right)
\end{aligned}
$$

where $\delta$ is a map of $D \times(-\varepsilon, \varepsilon)$ into $\widetilde{M}$ defined by $\delta(x, t):=f_{t}(x)$, and $\nabla^{t}$, $H_{t}$ and $\bar{B}^{t}$ are the quantities for $\left(f_{t}\right)_{c B}$. Since $\triangle_{\mathfrak{h}} v=\operatorname{div}_{\nabla} \bar{B}_{v}$, we have

$$
K\left(\widetilde{v}_{\nu}\right)\left(\theta^{\perp-1}(1)\right)=-\frac{1}{n+2 r} \triangle_{\mathfrak{h}} v
$$


Let $\left(\xi_{1}^{t}, \ldots, \xi_{r}^{t}\right)$ be a frame field of $N_{t}$ with $\theta_{t}^{\perp}\left(\xi_{1}^{t}, \ldots, \xi_{r}^{t}\right)=1$. Then we have

$$
\begin{aligned}
&\left.\frac{d}{d t}\right|_{t=0} K\left(\frac{d \nu_{t}}{d t}\right)\left(\theta_{t}^{\perp-1}(1)\right) \\
&=K\left(\left.\frac{d}{d t}\right|_{t=0} K\left(\frac{d \nu_{t}}{d t}\right)\right)\left(\theta^{\perp-1}(1)\right) \\
&+\sum_{\rho=1}^{r} K\left(\widetilde{v}_{\nu}\right)\left(\xi_{1}, \ldots,\left(\widetilde{\nabla}_{\left.\partial_{t}\right|_{t=0}} \xi_{\rho}^{t}\right)_{N}, \ldots, \xi_{r}\right) \\
&+\sum_{\rho=1}^{r} K\left(\widetilde{v}_{\nu}\right)\left(\xi_{1}, \ldots,\left(\widetilde{\nabla}_{\left.\partial_{t}\right|_{t=0}} \xi_{\rho}^{t}\right)_{T}, \ldots, \xi_{r}\right) \\
&= K\left(\left.\frac{d}{d t}\right|_{t=0} K\left(\frac{d \nu_{t}}{d t}\right)\right)\left(\theta^{\perp-1}(1)\right)-K\left(\widetilde{v}_{\nu}\right)\left(\theta^{\perp-1}(1)\right)^{2} \\
&+\sum_{\rho=1}^{r} K\left(\widetilde{v}_{\nu}\right)\left(\xi_{1}, \ldots, Q_{v}\left(\xi_{\rho}\right), \ldots, \xi_{r}\right) \\
&= K\left(\left.\frac{d}{d t}\right|_{t=0} K\left(\frac{d \nu_{t}}{d t}\right)\right)\left(\theta^{\perp-1}(1)\right)-K\left(\widetilde{v}_{\nu}\right)\left(\theta^{\perp-1}(1)\right)^{2} \\
&-\operatorname{tr}\left(Q_{v} \circ \nabla^{\perp} v\right) .
\end{aligned}
$$

Also, since $\left.\left(\delta_{*} \partial_{t}\right)_{T}\right|_{t=0}=v_{T}=0$, we have

$$
\begin{aligned}
& \left.\frac{d}{d t}\right|_{t=0} \operatorname{div}_{\nabla^{t}}\left(\delta_{*} \partial_{t}\right)_{T} \\
& =\left.\frac{d}{d t}\right|_{t=0}\left\{\sum_{i=1}^{n} \frac{\tau_{t}\left(\delta_{*} \partial_{1}, \ldots, \delta_{*}\left(\nabla_{\partial_{i}}^{t}\left(\delta_{*} \partial_{t}\right)_{T}\right), \ldots, \delta_{*} \partial_{n}\right)}{\tau_{t}\left(\delta_{*} \partial_{1}, \ldots, \delta_{*} \partial_{n}\right)}\right\} \\
& =\sum_{i=1}^{n} \frac{\tau\left(f_{*} \partial_{1}, \ldots,\left(\widetilde{\nabla}_{\left.\partial_{t}\right|_{t=0} \delta_{*}}\left(\nabla_{\partial_{i}}^{t}\left(\delta_{*} \partial_{t}\right)_{T}\right)\right)_{T}, \ldots, f_{*} \partial_{n}\right)}{\tau\left(f_{*} \partial_{1}, \ldots, f_{*} \partial_{n}\right)} \\
& =\sum_{i=1}^{n} \frac{\tau\left(f_{*} \partial_{1}, \ldots, f_{*} \nabla_{\partial_{i}}\left(\left(\widetilde{\nabla}_{\left.\partial_{t}\right|_{t=0}}\left(\delta_{*} \partial_{t}\right)_{T}\right)_{T}\right), \ldots, f_{*} \partial_{n}\right)}{\tau\left(f_{*} \partial_{1}, \ldots, f_{*} \partial_{n}\right)} \\
& =\operatorname{div}_{\nabla}\left(\widetilde{\nabla}_{\left.\partial_{t}\right|_{t=0}}\left(\delta_{*} \partial_{t}\right)_{T}\right)_{T},
\end{aligned}
$$

where $\tau_{t}$ is the tangential volume element map of $\left(f_{t}\right)_{c B}$. Also, since $H=0$, $\left.\left(\delta_{*} \partial_{t}\right)_{T}\right|_{t=0}=0$ and $\widetilde{M}$ is flat, we have

$$
\left.n \frac{d}{d t}\right|_{t=0} H\left(\left(\delta_{*} \partial_{t}\right)_{N}\right)
$$




$$
\begin{aligned}
= & \left.\frac{d}{d t}\right|_{t=0}\left(-\sum_{i=1}^{n} \frac{\tau_{t}\left(\delta_{*} \partial_{1}, \ldots, \widetilde{\nabla}_{\partial_{i}}\left(\left(\delta_{*} \partial_{t}\right)_{N}\right), \ldots, \delta_{*} \partial_{n}\right)}{\tau_{t}\left(\delta_{*} \partial_{1}, \ldots, \delta_{*} \partial_{n}\right)}\right) \\
= & -\sum_{i=1}^{n} \frac{K\left(\widetilde{v}_{\tau}\right)\left(f_{*} \partial_{1}, \ldots, \widetilde{\nabla}_{\partial_{i}} v, \ldots, f_{*} \partial_{n}\right)}{\theta\left(\partial_{1}, \ldots, \partial_{n}\right)} \\
& -\sum_{i=1}^{n} \sum_{j \neq i}\left(\left(A_{v}\right)_{j}^{j}\left(A_{v}\right)_{i}^{i}-\left(A_{v}\right)_{j}^{i}\left(A_{v}\right)_{i}^{j}\right) \\
& -\sum_{i=1}^{n} \frac{\tau\left(f_{*} \partial_{1}, \ldots, \widetilde{\nabla}_{\left.\partial_{t}\right|_{t=0}} \widetilde{\nabla}_{\partial_{i}}\left(\left(\delta_{*} \partial_{t}\right)_{N}\right), \ldots, f_{*} \partial_{n}\right)}{\theta\left(\partial_{1}, \ldots, \partial_{n}\right)} \\
& +\sum_{i=1}^{n} \frac{\tau\left(f_{*} \partial_{1}, \ldots, \widetilde{\nabla}_{\partial_{i}} v, \ldots, f_{*} \partial_{n}\right)}{\theta\left(\partial_{1}, \ldots, \partial_{n}\right)^{2}} \times\left.\frac{d}{d t}\right|_{t=0} \tau_{t}\left(\delta_{*} \partial_{1}, \ldots, \delta_{*} \partial_{n}\right) \\
= & n H(v) K\left(\widetilde{v}_{\tau}\right)\left(f_{*} \theta^{-1}(1)\right)-n^{2} H(v)^{2}+\operatorname{tr}\left(A_{v}^{2}\right) \\
& +\sum_{i=1}^{n} \frac{\tau\left(f_{*} \partial_{1}, \ldots, Q_{v}\left(\nabla_{\partial_{i}}^{\perp} v\right), \ldots, f_{*} \partial_{n}\right)}{\theta\left(\partial_{1}, \ldots, \partial_{n}\right)} \\
& -\sum_{i=1}^{n} \tau\left(f_{*} \partial_{1}, \ldots,-f_{*} A_{\left(\widetilde{\nabla}_{\partial_{t} \mid t=0}\left(\delta_{*} \partial_{t}\right)_{N}\right)_{N} \partial_{i}}\right. \\
& \left.+\nabla_{\partial_{i}}\left(\widetilde{\nabla}_{\left.\partial_{t}\right|_{t=0}}\left(\delta_{*} \partial_{t}\right)_{N}\right)_{T}, \ldots, f_{*} \partial_{n}\right) \\
& \times \frac{-n H(v)}{\theta\left(\partial_{1}, \ldots, \partial_{n}\right)}\left(K\left(\widetilde{v}_{\tau}\right)\left(f_{*} \theta^{-1}(1)\right)-n H(v)\right) \\
= & \operatorname{tr}\left(Q_{v} \circ \nabla^{\perp} v\right)+\operatorname{tr}\left(A_{v}^{2}\right)-\operatorname{div} \nabla\left(\widetilde{\nabla}_{\left.\partial_{t}\right|_{t=0}}\left(\delta_{*} \partial_{t}\right)_{N}\right)_{T},
\end{aligned}
$$

where $\left(A_{v}\right)_{i}^{j}$ is the component of $A_{v}$ with respect to $\left(\partial_{1}, \ldots, \partial_{n}\right)$. Also, since $H=0$ and $\widetilde{M}$ is flat, we have

$$
\begin{aligned}
\left.\frac{d}{d t}\right|_{t=0} & \operatorname{div}_{\nabla^{t}} \bar{B}_{\delta_{*} \partial_{t}}^{t} \\
= & \left.\frac{d}{d t}\right|_{t=0}\left(\sum_{i=1}^{n} \frac{\tau_{t}\left(\delta_{*} \partial_{1}, \ldots, \widetilde{\nabla}_{\partial_{i}} \delta_{*} \bar{B}_{\delta_{*} \partial_{t}}^{t}, \ldots, \delta_{*} \partial_{n}\right)}{\tau_{t}\left(\delta_{*} \partial_{1}, \ldots, \delta_{*} \partial_{n}\right)}\right) \\
= & \sum_{i=1}^{n} \frac{K\left(\widetilde{v}_{\tau}\right)\left(f_{*} \partial_{1}, \ldots, \widetilde{\nabla}_{\partial_{i}} f_{*} \bar{B}_{v}, \ldots, f_{*} \partial_{n}\right)}{\theta\left(\partial_{1}, \ldots, \partial_{n}\right)} \\
& -\sum_{i=1}^{n} \sum_{j \neq i}\left(\left(A_{v}\right)_{j}^{j}\left(\nabla \bar{B}_{v}\right)_{i}^{i}-\left(A_{v}\right)_{j}^{i}\left(\nabla \bar{B}_{v}\right)_{i}^{j}\right)
\end{aligned}
$$




$$
\begin{aligned}
& +\sum_{i=1}^{n} \frac{\tau\left(f_{*} \partial_{1}, \ldots, \widetilde{\nabla}_{\partial_{t} \mid t=0} \widetilde{\nabla}_{\partial_{i}} \delta_{*} \bar{B}_{\delta_{*} \partial_{t}}^{t}, \ldots, f_{*} \partial_{n}\right)}{\theta\left(\partial_{1}, \ldots, \partial_{n}\right)} \\
& +\sum_{i=1}^{n} \frac{-\tau\left(f_{*} \partial_{1}, \ldots, f_{*} \nabla_{\partial_{i}} \bar{B}_{v}, \ldots, f_{*} \partial_{n}\right)}{\theta\left(\partial_{1}, \ldots, \partial_{n}\right)^{2}} \\
& \times\left.\frac{d}{d t}\right|_{t=0} \tau_{t}\left(\delta_{*} \partial_{1}, \ldots, \delta_{*} \partial_{n}\right) \\
= & \left(\operatorname{div}_{\nabla} \bar{B}_{v}\right) K\left(\widetilde{v}_{\tau}\right)\left(f_{*} \theta^{-1}(1)\right)-n H(v) \operatorname{div}_{\nabla} \bar{B}_{v}+\operatorname{tr}\left(A_{v} \circ \nabla \bar{B}_{v}\right) \\
& -\sum_{i=1}^{n} \frac{\tau\left(f_{*} \partial_{1}, \ldots, Q_{v}\left(h\left(\bar{B}_{v}, \partial_{i}\right)\right), \ldots, f_{*} \partial_{n}\right)}{\theta\left(\partial_{1}, \ldots, \partial_{n}\right)} \\
& +\operatorname{div}_{\nabla}\left(\widetilde{\nabla}_{\left.\partial_{t}\right|_{t=0}} \delta_{*} \bar{B}_{\delta_{*} \partial_{t}}^{t}\right)_{T}-n H\left(\left(\widetilde{\nabla}_{\left.\partial_{t}\right|_{t=0}} \delta_{*} \bar{B}_{\delta_{*} \partial_{t}}^{t}\right)_{N}\right) \\
& -\operatorname{div}_{\nabla} \bar{B}_{v}\left(K\left(\widetilde{v}_{\tau}\right)\left(f_{*} \theta^{-1}(1)\right)-n H(v)\right) \\
= & \operatorname{tr}\left(A_{v} \circ \nabla \bar{B}_{v}\right)+\operatorname{div}_{\nabla}\left(\widetilde{\nabla}_{\left.\partial_{t}\right|_{t=0}} \delta_{*} \bar{B}_{\delta_{*} \partial_{t}}^{t}\right)_{T}-\operatorname{tr}\left(Q_{v} \circ h\left(\bar{B}_{v}, \cdot\right)\right) .
\end{aligned}
$$

From $(5.10) \sim(5.15)$, we have

$$
\begin{aligned}
K\left(\left.\frac{d}{d t}\right|_{t=0} K\left(\frac{d \nu_{t}}{d t}\right)\right)\left(\theta^{\perp-1}(1)\right) \\
=\frac{1}{n+2 r}\left\{\frac{1}{n+2 r}\left(\triangle_{\mathfrak{h}} v\right)^{2}+(n+2 r) \operatorname{tr}\left(Q_{v} \circ \nabla^{\perp} v\right)\right. \\
\quad+r \operatorname{div}_{\nabla}\left(\widetilde{\nabla}_{\left.\partial_{t}\right|_{t=0}}\left(\delta_{*} \partial_{t}\right)_{T}\right)_{T}-r \operatorname{tr}\left(Q_{v} \circ \nabla^{\perp} v\right) \\
\quad+\operatorname{tr}\left(Q_{v} \circ h\left(\bar{B}_{v}, \cdot\right)\right)-r \operatorname{tr}\left(A_{v}^{2}\right)+r \operatorname{div}_{\nabla}\left(\widetilde{\nabla}_{\left.\partial_{t}\right|_{t=0}}\left(\delta_{*} \partial_{t}\right)_{N}\right)_{T} \\
\left.\quad-\operatorname{tr}\left(A_{v} \circ \nabla \bar{B}_{v}\right)-\operatorname{div}_{\nabla}\left(\widetilde{\nabla}_{\left.\partial_{t}\right|_{t=0}} \delta_{*} \bar{B}_{\delta_{*} \partial_{t}}^{t}\right)_{T}\right\} .
\end{aligned}
$$

By substituting (5.11) and (5.16) into (5.9) and using the Green's theorem, we have the desired variational formula.

Let $f$ be a critical point of $\operatorname{Vol}_{c, D}$. Assume that there exists a parallel normal vector field $\bar{v}$ of $F$ such that $\mathfrak{h}(\bar{v})$ has the inverse, that is, the matrix $\left(\mathfrak{h}(\bar{v})^{i j}\right)$ consisting of the components of $\mathfrak{h}(\bar{v})$ is non-singular. Let $\eta$ be a positive function over $M$ whose support is contained in the closure $\bar{D}$ of $D$. Then we have the following second variational formula in terms of 
Theorem 5.7.

Corollary 5.8 Let $f_{t}(-\varepsilon<t<\varepsilon)$ be a transversal variation of $f$ in $\left.N D(M)_{\phi}\right|_{D}$ having $\eta \bar{v}$ as the variational vector field. Then we have

$$
\begin{aligned}
& \left.\frac{d^{2}}{d t^{2}}\right|_{t=0} \operatorname{Vol}_{c, D}\left(f_{t}\right) \\
& =-\frac{n+r}{n+2 r} \int_{D}\left\{\eta^{2} \operatorname{tr}\left(A_{\bar{v}}^{2}\right)+\frac{1}{n+2 r}\left(d \eta\left(\bar{L}^{-1}(\bar{v})\right)+\triangle_{\widehat{L}^{-1}(\bar{v})} \eta\right) \triangle_{\mathfrak{h}(\bar{v})} \eta\right. \\
& \left.\quad-\operatorname{tr}_{\widehat{L}^{-1}(\bar{v})}\left(\left(d \eta \circ A_{\bar{v}}\right) \otimes d \eta\right)\right\} \theta,
\end{aligned}
$$

where $A$ and $\mathfrak{h}$ are the quantities for $f_{c B}$ and $\widehat{L}^{-1}, \bar{L}^{-1}, \triangle_{\widehat{L}^{-1}(\bar{v})} \eta, \triangle_{\mathfrak{h}(\bar{v})} \eta$ and $\operatorname{tr}_{\widehat{L}^{-1}(\bar{v})}\left(\left(d \eta \circ A_{\bar{v}}\right) \otimes d \eta\right)$ are the quantities defined as below.

Remark 5.3 In the case where $r=1$ and $\bar{v}$ is the affine normal of $f$, we have $L_{1 i}^{j 1}=\left(L^{-1}\right)_{i 1}^{1 j}=\delta_{i j},\left(\widehat{L}^{-1}\right)_{1}^{i j}=\mathfrak{h}^{i j}$ and $\left(\bar{L}^{-1}\right)^{i}=\bar{\nabla}_{j} \mathfrak{h}^{i j}=T^{i}=0$, where $\left(\mathfrak{h}^{i j}\right)=\left(h_{i j}\right)^{-1}$. Hence this second variational formula is as follows:

$$
\begin{aligned}
\left.\frac{d^{2}}{d t^{2}}\right|_{t=0} \operatorname{Vol}_{c, D}\left(f_{t}\right)=- & \frac{n+1}{n+2} \int_{D}\left\{\eta^{2} \operatorname{tr}\left(A_{\bar{v}}^{2}\right)+\frac{1}{n+2}\left(\triangle_{\mathfrak{h}} \eta\right)^{2}\right. \\
& \left.\left.-\operatorname{tr}_{h}\left(\left(d \eta \circ A_{\bar{v}}\right) \otimes d \eta\right)\right)\right\} \theta
\end{aligned}
$$

which coincides with the second variational formula in Theorem 4.2 of $[\mathrm{VeVr}]$.

Let $h$ and $\mathfrak{h}$ be as in Corollary 5.8. Define a section $L$ of $N^{*} \otimes T M \otimes$ $T^{*} M \otimes N$ by $L_{\rho i}^{j \mu}:=\mathfrak{h}_{\rho}^{j k} h_{k i}^{\mu}$, where $\mathfrak{h}_{\rho}^{i j}$ and $h_{k i}^{\mu}$ are the components of $\mathfrak{h}$ and $h$ with respect to $\left(\partial_{1}, \ldots, \partial_{n}\right)$ and $\left(\xi_{1}, \ldots, \xi_{r}\right)$ in the previous paragraph. Note that the following matrix is non-singular: 


$$
\mathfrak{L}:=\left(\begin{array}{ccccccc}
L_{11}^{11} & \cdots & L_{11}^{1 r} & \cdots & L_{1 n}^{11} & \cdots & L_{1 n}^{1 r} \\
\vdots & & \vdots & & \vdots & & \vdots \\
L_{r 1}^{11} & \cdots & L_{r 1}^{1 r} & \cdots & L_{r n}^{11} & \cdots & L_{r n}^{1 r} \\
\vdots & & \vdots & & \vdots & & \vdots \\
L_{11}^{n 1} & \cdots & L_{11}^{n r} & \cdots & L_{1 n}^{n 1} & \cdots & L_{1 n}^{n r} \\
\vdots & & \vdots & & \vdots & & \vdots \\
L_{r 1}^{n 1} & \cdots & L_{r 1}^{n r} & \cdots & L_{r n}^{n 1} & \cdots & L_{r n}^{n r}
\end{array}\right)
$$

Hence a section $L^{-1}$ of $N \otimes T^{*} M \otimes T M \otimes N^{*}$ is defined by $\left(L^{-1}\right)_{i \mu}^{\rho j} L_{\rho k}^{i \nu}=$ $\delta_{\mu}^{\nu} \delta_{k}^{j}$, where $\delta_{\mu}^{\nu}$ and $\delta_{k}^{j}$ are the Kronecker's delta. Furthermore, we define a section $\widehat{L}^{-1}$ of $N^{*} \otimes T M \otimes T M$ and a section $\bar{L}^{-1}$ of $N^{*} \otimes T M$ by $\left(\widehat{L}^{-1}\right)_{\rho}^{i j}:=\left(L^{-1}\right)_{k \rho}^{\mu i} \mathfrak{h}_{\mu}^{k j}$ and $\left(\bar{L}^{-1}\right)_{\rho}^{i}:=\bar{\nabla}_{j}\left(\widehat{L}^{-1}\right)_{\rho}^{i j}$, respectively, where $\bar{\nabla}$ is the connection of $N^{*} \otimes T M \otimes T M$ induced from the induced connection $\nabla$ on $M$ and the transversal connection $\nabla^{\perp}$. For a function $\eta$ on $M$ and a parallel transevrsal vector field $\bar{v}$, we define $\triangle_{\mathfrak{h}(\bar{v})} \eta$ and $\triangle_{\widehat{L}^{-1}(\bar{v})} \eta$ by $\triangle_{\mathfrak{h}(\bar{v})} \eta:=\mathfrak{h}(\bar{v})^{i j}\left(\nabla^{2} \eta\right)_{i j}$ and $\triangle_{\widehat{L}^{-1}(\bar{v})} \eta:=\widehat{L}^{-1}(\bar{v})^{i j}\left(\nabla^{2} \eta\right)_{i j}$, respectively. Also, we define $\operatorname{tr}_{\widehat{L}^{-1}(\bar{v})}\left(\left(d \eta \circ A_{\bar{v}}\right) \otimes d \eta\right)$ by $\left.\operatorname{tr}_{\widehat{L}^{-1}(\bar{v})}\left(\left(d \eta \circ A_{\bar{v}}\right) \otimes d \eta\right)\right):=$ $\widehat{L}^{-1}(\bar{v})^{i j}\left(d \eta \circ A_{\bar{v}}\right)_{i} d \eta_{j}$.

Proof of Corollary 5.8. Let $v:=\eta \bar{v}$. According to Theorem 5.7, we have

$$
\begin{aligned}
& \left.\frac{d^{2}}{d t^{2}}\right|_{t=0} \operatorname{Vol}_{c, D}\left(f_{t}\right) \\
& =\frac{-1}{n+2 r} \int_{D}\left\{(n+r) \eta^{2} \operatorname{tr}\left(A_{\bar{v}}^{2}\right)-\frac{1}{n+2 r}\left(\triangle_{\mathfrak{h}} v\right)^{2}-\operatorname{tr}\left(A_{v} \circ \nabla \bar{B}_{v}\right)\right. \\
& \left.\quad+(n+r) \operatorname{tr}\left(Q_{v} \circ \nabla^{\perp} v\right)+\operatorname{tr}\left(Q_{v} \circ h\left(\bar{B}_{v}, \cdot\right)\right)\right\} \theta
\end{aligned}
$$

By somewhat long calculations, we have

$$
\triangle_{\mathfrak{h}} v=\operatorname{div}_{\nabla} \bar{B}_{v}=\triangle_{\mathfrak{h}(\bar{v})} \eta
$$

and

$$
\begin{aligned}
& \operatorname{tr}\left(A_{v} \circ \nabla \bar{B}_{v}\right) \\
& \quad=\operatorname{div}_{\nabla}\left(\eta \operatorname{tr}_{\mathfrak{h}(\bar{v})}\left(d \eta \otimes A_{\bar{v}}\right)\right)-\operatorname{tr}_{\mathfrak{h}(\bar{v})}\left(\left(d \eta \circ A_{\bar{v}}\right) \otimes d \eta\right),
\end{aligned}
$$

where we use the Codazzi equation. Let $\delta: D \times(-\varepsilon, \varepsilon) \rightarrow \widetilde{M}$ by $\delta(x, t)=$ 
$f_{t}(x) \quad((x, t) \in D \times(-\varepsilon, \varepsilon))$ and $N_{t}$ (resp. $\theta_{t}^{\perp}$ ) be the transversal bundle (resp. the transversal volume element) of $\left(f_{t}\right)_{c B}$. Take a local coordinate $\left(x^{1}, \ldots, x^{n}\right)$ on an open set $U$ of $D$ and vector fields $\xi_{1}, \ldots, \xi_{r}$ along $\left.\delta\right|_{U \times(-\varepsilon, \varepsilon)}$ such that $\left(\xi_{1}\right)_{(x, t)}, \ldots,\left(\xi_{r}\right)_{(x, t)}$ give a frame of $N_{t}$ with $\theta_{t}^{\perp}\left(\left(\xi_{1}\right)_{(x, t)}, \ldots,\left(\xi_{r}\right)_{(x, t)}\right)=1$ for each $(x, t) \in U \times(-\varepsilon, \varepsilon)$. Let $h_{i j}^{\rho},\left(Q_{v}\right)_{\rho}^{i}$ and $\left(A_{\bar{v}}\right)_{i}^{j}$ be the components of $h, Q_{v}$ and $A_{\bar{v}}$ with respect to $\left(\partial_{1}, \ldots, \partial_{n}\right)$ and $\left(\xi_{1}\right)_{(\cdot, 0)}, \ldots,\left(\xi_{r}\right)_{(\cdot, 0)}$, where $\partial_{i}:=\partial / \partial x^{i}$. Let $\left(\widetilde{\nabla}_{\left.\partial_{t}\right|_{t=0}} \xi_{\rho}\right)_{N}=\sum_{\mu=1}^{r} \kappa_{\rho}^{\mu} \xi_{\mu}$. Then, by somewhat long calculations, we have

$$
h_{i j}^{\rho}\left(Q_{v}\right)_{\rho}^{j}=-\partial_{i} \kappa_{\rho}^{\rho}-\left(A_{\bar{v}}\right)_{i}^{j} \partial_{j} \eta .
$$

By operating $\mathfrak{h}_{\mu}^{k i}$ to both sides of this relation, we have

$$
L_{\mu j}^{k \rho}\left(Q_{v}\right)_{\rho}^{j}=-\mathfrak{h}_{\mu}^{k i}\left(\partial_{i} \kappa_{\rho}^{\rho}+\left(A_{\bar{v}}\right)_{i}^{j} \partial_{j} \eta\right) .
$$

Furthermore, by operating $\left(L^{-1}\right)_{k \nu}^{\mu l}$ to both sides of this relation, we have

$$
\left(Q_{v}\right)_{\nu}^{l}=-\left(\widehat{L}^{-1}\right)_{\nu}^{l i}\left(\partial_{i} \kappa_{\rho}^{\rho}+\left(A_{\bar{v}}\right)_{i}^{j} \partial_{j} \eta\right) .
$$

Hence we have

$$
\begin{aligned}
& \operatorname{tr}\left(Q_{v} \circ \nabla^{\perp} v\right)=\left(\partial_{i} \eta\right)\left(Q_{v}\right)_{\rho}^{i} \bar{v}^{\rho} \\
& \quad=-\operatorname{tr}_{\widehat{L}^{-1}(\bar{v})}\left(d \eta \otimes d\left(\kappa_{\rho}^{\rho}\right)\right)-\operatorname{tr}_{\widehat{L}^{-1}(\bar{v})}\left(\left(d \eta \circ A_{\bar{v}}\right) \otimes d \eta\right) .
\end{aligned}
$$

Also, from (5.20), we have

$$
\begin{aligned}
& \operatorname{tr}\left(Q_{v} \circ h\left(\bar{B}_{v}, \cdot\right)\right)=\left(\partial_{i} \eta\right) \mathfrak{h}(\bar{v})^{j i} h_{j k}^{\rho}\left(Q_{v}\right)_{\rho}^{k} \\
& \quad=-\operatorname{tr}_{\mathfrak{h}(\bar{v})}\left(d \eta \otimes d\left(\kappa_{\rho}^{\rho}\right)\right)-\operatorname{tr}_{\mathfrak{h}(\bar{v})}\left(\left(d \eta \circ A_{\bar{v}}\right) \otimes d \eta\right) .
\end{aligned}
$$

On the other hand, it follows from $\nabla^{\perp} \bar{v}=0$ that

$$
\operatorname{tr}_{\mathfrak{h}(\bar{v})}\left(d \eta \otimes d\left(\kappa_{\rho}^{\rho}\right)\right)=\operatorname{div}_{\nabla} X-\left(\triangle_{\mathfrak{h}(\bar{v})} \eta\right) \kappa_{\rho}^{\rho},
$$

that is,

$$
\begin{aligned}
& \operatorname{tr}\left(Q_{v} \circ h\left(\bar{B}_{v}, \cdot\right)\right) \\
& \quad=-\operatorname{div}_{\nabla} X+\left(\triangle_{\mathfrak{h}(\bar{v})} \eta\right) \kappa_{\rho}^{\rho}-\operatorname{tr}_{\mathfrak{h}(\bar{v})}\left(\left(d \eta \circ A_{\bar{v}}\right) \otimes d \eta\right),
\end{aligned}
$$

where $X$ is defined by $X^{i}:=\mathfrak{h}(\bar{v})^{i j}\left(\partial_{j} \eta\right) \kappa_{\rho}^{\rho}$. Also, we have

$$
\begin{aligned}
& \operatorname{tr}_{\widehat{L}^{-1}(\bar{v})}\left(d \eta \otimes d\left(\kappa_{\rho}^{\rho}\right)\right) \\
& \quad=\operatorname{div}_{\nabla} Y-\bar{L}^{-1}(\bar{v})^{i}\left(\partial_{i} \eta\right) \kappa_{\rho}^{\rho}-\left(\triangle_{\widehat{L}^{-1}(\bar{v})} \eta\right) \kappa_{\rho}^{\rho},
\end{aligned}
$$


where $Y$ is defined by $Y^{i}:=\widehat{L}^{-1}(\bar{v})^{j i}\left(\partial_{j} \eta\right) \kappa_{\rho}^{\rho}$. From (5.22) and (5.25), we have

$$
\begin{aligned}
\operatorname{tr}\left(Q_{v} \circ \nabla^{\perp} v\right)=- & \operatorname{div}_{\nabla} Y+\bar{L}^{-1}(\bar{v})^{i}\left(\partial_{i} \eta\right) \kappa_{\rho}^{\rho}+\left(\triangle_{\widehat{L}^{-1}(\bar{v})} \eta\right) \kappa_{\rho}^{\rho} \\
& -\operatorname{tr}_{\widehat{L}^{-1}(\bar{v})}\left(\left(d \eta \circ A_{\bar{v}}\right) \otimes d \eta\right) .
\end{aligned}
$$

On the other hand, by somewhat long calculations, we can show

$$
\begin{aligned}
& \left(\partial_{i} \partial_{j} \eta\right) \bar{v}^{\rho}-\eta h_{i k}^{\rho}\left(A_{\bar{v}}\right)_{j}^{k} \\
& \quad=\left(\left(\nabla_{\partial_{i}} \partial_{j}\right) \eta\right) \bar{v}^{\rho}+\left.\partial_{t}\right|_{t=0}\left(h_{t}\right)_{i j}^{\rho}+h_{i j}^{\mu} \kappa_{\mu}^{\rho},
\end{aligned}
$$

where we use the flatness of $\widetilde{\nabla}$. By operating $\mathfrak{h}_{\rho}^{i j}$ to this both sides, we have

$$
\triangle_{\mathfrak{h}(\bar{v})} \eta=n \kappa_{\rho}^{\rho}+2 r \frac{\left.\partial_{t}\right|_{t=0} \omega_{h_{t}}}{\omega_{h}}
$$

where we use $H(\bar{v})=0$. Also, from (5.4), (5.7) and (5.18), we have

$$
\left.\partial_{t}\right|_{t=0} \omega_{h_{t}}\left(\partial_{1}, \ldots, \partial_{n}\right)=\frac{1}{n+2 r}\left(\triangle_{\mathfrak{h}(\bar{v})} \eta\right) \omega_{h}\left(\partial_{1}, \ldots, \partial_{n}\right)
$$

that is, $\left.\partial_{t}\right|_{t=0} \omega_{h_{t}} / \omega_{h}=1 /(n+2 r) \triangle_{\mathfrak{h}(\bar{v})} \eta$. Hence we have $\kappa_{\rho}^{\rho}=1 /(n+$ $2 r) \triangle_{\mathfrak{h}(\bar{v})} \eta$. By substituting this relation, (5.18), (5.19), (5.24) and (5.26) into (5.17) and using the Green's theorem, we can obtain the desired relation.

From this corollary, we directly have the following fact.

Corollary 5.9 Let $f$ be an immersion of an $n$-dimensional manifold $M$ into an $(n+r)$-dimensional flat equiaffine manifold $(\widetilde{M}, \widetilde{\nabla}, \widetilde{\theta})$ and $D$ be a non-degenerate part of $f$. Assume that $D$ has a relatively compact component $D_{0}$ with smooth boundary. Let $f_{t}(-\varepsilon<t<\varepsilon)$ be a transversal variation of $f$ satisfying $\left.f_{t}\right|_{M \backslash D_{0}}=\left.f\right|_{M \backslash D_{0}}$ and $\nabla^{\perp} v=0$, where $v$ is a variational vector field of $f_{t}$ and $\nabla^{\perp}$ is the transeversal connection of $\left(\left.f\right|_{D_{0}}\right)_{c B}$. If the affine mean curvature function $H$ of $\left(\left.f\right|_{D_{0}}\right)_{c B}$ vanishes and the affine shape operator $A_{v}$ of $\left(\left.f\right|_{D_{0}}\right)_{c B}$ is non-zero and real diagonalizable, then $\left.f\right|_{D_{0}}$ is a critical point of $\operatorname{Vol}_{c, D_{0}}$ and $\left.\frac{d^{2}}{d t^{2}}\right|_{t=0} \operatorname{Vol}_{c, D_{0}}\left(\left.f_{t}\right|_{D_{0}}\right)<0$, that is, the critical point $\left.f\right|_{D_{0}}$ is not a minimal point of $\operatorname{Vol}_{c, D_{0}}$.

Remark 5.4 Since $\partial D_{0}$ consists of degenerate points of $f$, there is possible to exist a $\nabla^{\perp}$-parallel transversal vector field $v$ on $D_{0}$ satisfying $\lim _{k \rightarrow \infty} v_{x_{k}}=0_{x_{0}}$ for any sequence $\left\{x_{k}\right\}_{k=1}^{\infty}$ in $D_{0}$ with $\lim _{k \rightarrow \infty} x_{k} \in \partial D_{0}$, 

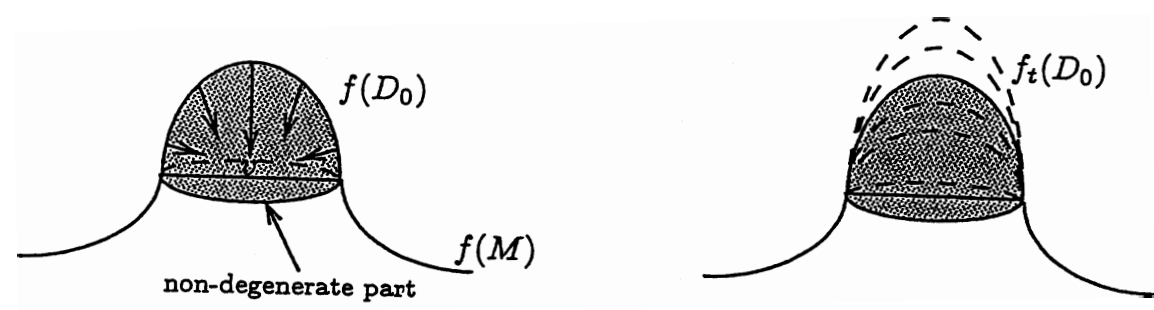

Fig. 4.

where $x_{0}:=\lim _{k \rightarrow \infty} x_{k}$ and $0_{x_{0}}$ is the zero element of $N_{x_{0}}$. In particular, if $r=1$, then any $\nabla^{\perp}$-parallel transversal vector field on $D_{0}$ is such one. Hence, there is possible to exist the transversal variation $f_{t}$ as in the statement of this corollary.

Proof. According to Corollary 5.5, $\left.f\right|_{D_{0}}$ is a critical point of $\mathrm{Vol}_{c, D_{0}}$ because of $H=0$. Hence, it follows from Corollary 5.8 that

$$
\left.\frac{d^{2}}{d t^{2}}\right|_{t=0} \operatorname{Vol}_{c, D_{0}}\left(\left.f_{t}\right|_{D_{0}}\right)=-\frac{n+r}{n+2 r} \int_{D_{0}} \operatorname{tr}\left(A_{v}^{2}\right) \theta
$$

where we use $\nabla^{\perp} v=0$. Since $A_{v}$ is non-zero and real diagonalizable, we have $\operatorname{tr}\left(A_{v}^{2}\right)>0$ and hence $\left.\frac{d^{2}}{d t^{2}}\right|_{t=0} \operatorname{Vol}_{c, D_{0}}\left(\left.f_{t}\right|_{D_{0}}\right)<0$. Thus $\left.f\right|_{D_{0}}$ is not a minimal point of $\mathrm{Vol}_{c, D_{0}}$.

\section{References}

[C] Calabi E., Hypersurfaces with maximal affinely invariant area. Amer. J. Math. 104 (1982), 91-126.

[DMVV] Dillen F., Mys G., Verstraelen L. and Vrancken L., The affine mean curvature vector for surfaces in $\mathbf{R}^{4}$. Math. Nachr. 166 (1994), 155-165.

[K] Koike N., The Lipschitz-Killing curvature for an equiaffine immersion and theorems of Gauus-Bonnet type and Chern-Lashof type. Result. Math. 39 (2001), 230-244.

[KT] Koike N. and Takekuma K., Equiaffine immersions of general codimension and its transversal volume element map. Result. Math. 39 (2001), 274-291.

[NSa] Nomizu K. and Sasaki T., Affine differential geometry, Cambridge University Press, 1994.

[Sa1] Sasaki T., On the Veronese embedding and related system of differential equations. Lecture Notes in Math. 1481, Springer-Verlag, New York, 1991, pp. $210-247$. 
[Sa2] Sasaki T., Affine immersion of $n$-dimensional manifold into $\boldsymbol{R}^{n+n(n+1) / 2}$ and affine minimality. Geometriae Dedicata 57 (1995), 317-333.

[VeVr] Verstraelen L. and Vrancken L., Affine variation formulas and affine minimal surfaces. Michigan Math. J. 36 (1989), 77-93.

[We] Weise K.H., Der Berührungstensor zweiter Flächen und die Affingeometrie der $F_{p}$ im $A_{n}$. Math. Z. 43 (1938), 469-480, 44 (1939), 161-184.

[Wi] Wiehe M., A unimodularly invariant theory for immersions into the affine space. Math. Z. 241 (2002), 353-373.

Department of Mathematics, Faculty of Science Tokyo University of Science

26 Wakamiya-cho Shinjuku-ku

Tokyo 162-8601 Japan

E-mail: koike@ma.kagu.tus.ac.jp 